\title{
On the nonlinear Neumann problem involving the critical Sobolev exponent and Hardy potential
}

\author{
Jan CHABROWSKI \\ Department of Mathematics, \\ University of Queensland \\ St. Lucia 4072, QId, Australia \\ jhc@maths . uq. edu . au
}

Recibido: 9 de Septiembre de 2003

Aceptado: 30 de Octubre de 2003

\begin{abstract}
In this paper we investigate the solvability of the Neumann problems (1), (12), (16), (32) and (43) involving the critical Sobolev and Hardy exponents. It is assumed that the coefficient $Q$ is a positive and smooth function on $\bar{\Omega}, \mu$ and $\lambda$ are real parameters. We examine the common effect of the mean curvature of the boundary $\partial \Omega$, the shape of the graph of the coefficient $Q$ and the singular Hardy potential on the existence and the nonexistence of solutions of these problems.
\end{abstract}

Key words: Neumann problem, critical Sobolev exponent, singular Hardy potential, least energy solutions, topological linking

2000 Mathematics Subject Classification: 35B33, 35J20, 35J65

\section{Introduction}

In this paper we investigate the nonlinear elliptic problem involving the Neumann conditions

$$
\begin{cases}-\Delta u+\frac{\mu}{|x|^{2}} u & =Q(x)|u|^{2^{*}-2} u \quad \text { in } \Omega, \\ \frac{\partial}{\partial \nu} u(x) & =0 \quad \text { on } \partial \Omega,\end{cases}
$$

where the coefficient $Q$ is continuous and positive on $\bar{\Omega}, \mu$ is a real parameter, $\nu$ is an outward normal to the boundary $\partial \Omega$ and $2^{*}=\frac{2 N}{N-2}, N \geq 3$, is a critical Sobolev exponent. We assume that $0 \in \Omega$ and that $\Omega$ is a bounded domain in $\mathbb{R}^{N}$ with a smooth boundary. We also study a more general problem (12) (see Section 3) with 
an additional term $\lambda u$. In Section 4 we extend this to problem (16), obtained from (12) by replacing $\mu$ with $-\mu, \mu>0$. In Sections 7 and 8 the term $\frac{\mu}{|x|^{2}} u$ is replaced by $\frac{\mu}{|x|_{\alpha}^{2 *}}|u|^{2_{\alpha}^{*}-2} u$, where $2_{\alpha}^{*}=\frac{2(N-\alpha)}{N-2}, 0<\alpha<2$ is the critical Hardy-Sobolev exponent.

In recent years the nonlinear Neumann problem involving critical Sobolev exponent has been widely studied in [3], [4], [5], [7], [8], [9]. In these papers the existence of least energy solutions has been established for problem (1), with the singular term $\mu \frac{u}{|x|^{2}}$ replaced by $\lambda u, \lambda>0$ and with $Q(x)=1$ on $\Omega$. Further extensions of these results to the problem with $Q(x) \neq$ constant can be found in [13], [14], [15]. The novelty here is that we consider the Neumann problem involving the singular potential $\frac{1}{|x|^{2}}$ and the critical Sobolev exponent. Equation (1) with the Dirichlet boundary conditions, has been studied in [1], [16], [23] and [20]. The singular potential $\frac{1}{|x|^{2}}$ is related to the Hardy inequality. We recall the classical Hardy inequality (known also as the Uncertainty Principle): if $u \in H_{\circ}^{1}(\Omega)$, then $\frac{u}{|x|} \in L^{2}(\Omega)$ and

$$
\int_{\Omega} \frac{u^{2}}{|x|^{2}} d x \leq c_{N} \int_{\Omega}|\nabla u|^{2} d x
$$

where $c_{N}=\frac{4}{(N-2)^{2}}$ and this constant is optimal. It is also known that the constant $\frac{1}{c_{N}}$ is not achieved. Therefore one can expect an error term on the left side of this inequality. Some estimates of this error term can be found in the papers [2], [12] and [24]. Problem (1) has a variational structure and the underlying Sobolev space for $(1)$ is $H^{1}(\Omega)$. Since this space contains constant functions, it is clear that this inequality is no longer true in $H^{1}(\Omega)$. In Section 2 we give a suitable modification of (2) which will be used in this paper. In Sections 3 and 4 we investigate the existence of the least energy solutions. Section 6 is devoted to the case where a parameter $\lambda$ in interferes with the spectra of $-\Delta+\frac{\mu}{|x|^{2}}$ and $-\Delta-\frac{\mu}{|x|^{2}}$. Our approach is based on a min-max principle involving the topological linking [36]. Sections 7 and 8 are devoted to nonlinear Neumann problems involving the critical Hardy-Sobolev exponent. We establish the existence of solutions through the mountain-pass principle.

We recall that a $C^{1}$ functional $\phi: X \rightarrow \mathbb{R}$ on a Banach space $X$ satisfies the PalaisSmale condition at level $c\left((P S)_{c}\right.$ condition for short), if each sequence $\left\{x_{n}\right\} \subset X$ such that $(*) \phi\left(x_{n}\right) \rightarrow c$ and $(* *) \phi^{\prime}\left(x_{n}\right) \rightarrow 0$ in $X^{*}$ is relatively compact in $X$. Finally, any sequence $\left\{x_{n}\right\}$ satisfying $(*)$ and $(* *)$ is called a Palais-Smale sequence at level $c$ $\left(\mathrm{a}(P S)_{c}\right.$ sequence for short).

Throughout this paper we denote strong convergence by " $\rightarrow$ " and weak convergence by "- - ". The norms in the Lebesgue spaces $L^{p}(\Omega)$ are denoted by $\|\cdot\|$. By $H^{1}(\Omega)$ we denote a standard Sobolev space on $\Omega$ equipped with norm

$$
\|u\|^{2}=\int_{\Omega}\left(|\nabla u|^{2}+u^{2}\right) d x
$$




\section{Palais-Smale condition in the case $\mu>0$}

Throughout this and the next section, we assume that $\mu>0$. We commence by extending the Hardy inequality to the space $H^{1}(\Omega)$.

Lemma 2.1. For every $\delta>0$ there exists a constant $C=C(\delta,|\Omega|)>0$ such that

$$
\int_{\Omega} \frac{u^{2}}{|x|^{2}} d x \leq\left(c_{N}+\delta\right) \int_{\Omega}|\nabla u|^{2} d x+C(\delta,|\Omega|) \int_{\Omega} u^{2} d x
$$

for every $u \in H^{1}(\Omega)$.

Proof. Let $\rho>0$ be such that $\overline{B(0,2 \rho)} \subset \Omega$. We define a $C^{1}$-function $\phi$ such that $\phi(x)=1$ on $B(0, \rho), 0 \leq \phi(x) \leq 1$ on $\Omega$ and $\phi(x)=0$ on $\Omega-B(0, \rho)$. It then follows from (2) that

$$
\begin{aligned}
\int_{\Omega} \frac{u^{2}}{|x|^{2}} d x & =\int_{\Omega} \frac{(\phi u)^{2}}{|x|^{2}} d x+\int_{\Omega} \frac{u^{2}}{|x|^{2}}\left(1-\phi^{2}\right) d x \\
& \leq c_{N} \int_{\Omega}|\nabla(\phi u)|^{2} d x+\int_{\Omega} \frac{u^{2}\left(1-\phi^{2}\right)}{|x|^{2}} d x
\end{aligned}
$$

Applying the Young inequality, we get

$$
\int_{\Omega} \frac{u^{2}}{|x|^{2}} d x \leq\left(c_{N}+\delta\right) \int_{\Omega}|\nabla u|^{2} \phi^{2} d x+\int_{\Omega} u^{2}\left(c_{N}|\nabla \phi|^{2}+\frac{c_{N}^{2}}{\delta}|\nabla \phi|^{2}+\frac{1-\phi^{2}}{|x|^{2}}\right) d x
$$

and the result follows.

One can define the best Hardy constant in $H^{1}(\Omega)$ by

$$
S_{h}=\inf _{u \in H^{1}(\Omega)} \frac{\int_{\Omega}\left(|\nabla u|^{2}+u^{2}\right) d x}{\int_{\Omega} \frac{u^{2}}{|x|^{2}} d x} .
$$

The constant $S_{h}$ depends on $|\Omega|$ and tends to 0 as $|\Omega| \rightarrow 0$. Since $\Omega$ is a bounded domain, it follows from Lemma 2.1 that

$$
\|u\|^{2}=\int_{\Omega}\left(|\nabla u|^{2}+\frac{u^{2}}{|x|^{2}}\right) d x
$$

defines an equivalent norm on $H^{1}(\Omega)$.

We set

$$
Q_{m}=\max _{x \in \partial \Omega} Q(x) \quad \text { and } \quad Q_{M}=\max _{x \in \bar{\Omega}} Q(x) .
$$

Let

$$
S_{\infty}=\min \left(\frac{S}{2^{\frac{2}{N}} Q_{m^{\frac{N-2}{N}}}^{\frac{N-2}{N}}}\right),
$$


where $S$ is the best Sobolev constant defined by

$$
S=\inf \left\{\int_{\mathbb{R}^{N}}|\nabla u|^{2} d x ; u \in D^{1,2}\left(\mathbb{R}^{N}\right), \int_{\mathbb{R}^{N}}|u|^{2^{*}} d x\right\} .
$$

Here $D^{1,2}\left(\mathbb{R}^{N}\right)$ denotes the Sobolev space obtained as the completion of $C_{\circ}^{\infty}\left(\mathbb{R}^{N}\right)$ with respect to the norm

$$
\|u\|_{D^{1,2}\left(\mathbb{R}^{N}\right)}^{2}=\int_{\mathbb{R}^{N}}|\nabla u|^{2} d x
$$

To find a solution of (1) we consider the constrained variational problem

$$
S_{\mu}=\inf \left\{\int_{\Omega}\left(|\nabla u|^{2}+\frac{\mu}{|x|^{2}} u^{2}\right) d x ; u \in H^{1}(\Omega), \int_{\Omega} Q(x)|u|^{2^{*}} d x=1\right\} .
$$

By Lemma 2.1 and the Sobolev inequality, we see that $0<S_{\mu}<\infty$ for every $\mu>0$. If $u$ is a minimizer for $S_{\mu}$, then $S_{\mu}^{\frac{1}{2^{*}-2}} u$ is a solution of (1).

Proposition 2.2. If $S_{\mu}<S_{\infty}$ for some $\mu>0$, then $S_{\mu}$ has a minimizer.

Proof. The proof is standard and relies on P. L. Lions' concentration-compactness principle [25]. Let $\left\{u_{m}\right\}$ be a minimizing sequence for $S_{\mu}$. Since $\left\{u_{m}\right\}$ is bounded in $H^{1}(\Omega)$, we may assume that $u_{m} \rightarrow u$ in $H^{1}(\Omega)$ and $L^{2^{*}}(\Omega)$ and $u_{m} \rightarrow u$ in $L^{p}(\Omega)$ for $2 \leq p<2^{*}$. By the concentration-compactness principle, we may assume that

$$
\left|u_{m}\right|^{2^{*}} \stackrel{*}{\rightarrow}|u|^{2^{*}}+\sum_{j \in J} \nu_{j} \delta_{x_{j}} \quad \text { and } \quad\left|\nabla u_{m}\right|^{2} \stackrel{*}{\rightarrow}|\nabla u|^{2}+\sum_{j \in J} \mu_{j} \delta_{x_{j}},
$$

in the sense of measure, where $\nu_{j}>0, \mu_{j}>0$ are constants and the set $J$ is at most countable. Moreover, we have

$$
\text { if } x_{j} \in \Omega \text {, then } S \nu_{j}^{\frac{2}{2^{*}}} \leq \mu_{j}
$$

and

$$
\text { if } x_{j} \in \partial \Omega \text {, then } S \frac{\nu_{j}^{\frac{2}{2^{*}}}}{2^{\frac{2}{N}}} \leq \mu_{j}
$$

The only possible concentration point for $\left\{\frac{u_{m}^{2}}{|x|^{2}}\right\}$ is 0 . However, if this occurs, then $\left\{\left|\nabla u_{m}\right|^{2}\right\}$ also concentrates at 0 . Hence it is sufficient to show that $\mu_{j}=\nu_{j}=0$ for all $j \in J$. We write

$$
1=\int_{\Omega} Q(x)|u|^{2^{*}} d x+\sum_{j \in J} Q\left(x_{j}\right) \nu_{j}
$$


We also have

$$
\begin{aligned}
S_{\mu} \geq & \int_{\Omega}\left(|\nabla u|^{2}+\frac{\mu}{|x|^{2}} u^{2}\right) d x+\sum_{j \in J} \mu_{j} \\
\geq & S_{\mu}\left(\int_{\Omega} Q(x)|u|^{2^{*}} d x\right)^{\frac{2}{2^{*}}}+\sum_{x_{j} \in \Omega} S \nu_{j}^{\frac{2}{2^{*}}}+\sum_{x_{j} \in \partial \Omega} \frac{S}{2^{\frac{2}{N}}} \nu_{j}^{\frac{2}{2^{*}}} \\
\geq & S_{\mu}\left(\int_{\Omega} Q(x)|u|^{2^{*}} d x\right)^{\frac{2}{2^{*}}}+\sum_{x_{j} \in \Omega} \frac{S}{Q_{M}^{\frac{N-2}{N}}}\left(\nu_{j} Q\left(x_{j}\right)\right)^{\frac{N-2}{N}} \\
& +\sum_{x_{j} \in \partial \Omega} \frac{S}{Q_{m^{\frac{N-2}{N}}}^{\frac{2}{N}}}\left(\nu_{j} Q\left(x_{j}\right)\right)^{\frac{N-2}{N}} .
\end{aligned}
$$

Since $S_{\mu}<S_{\infty}$, we deduce from (5) that $\nu_{j}=0$ for every $j \in J$.

To estimate $S_{\mu}$, we test the functional

$$
E_{\mu, Q}(u)=\frac{\int_{\Omega}\left(|\nabla u|^{2}+\frac{\mu}{|x|^{2}} u^{2}\right) d x}{\left(\int_{\Omega} Q(x)|u|^{2^{*}} d x\right)^{\frac{2}{2^{*}}}}
$$

with instantons. We recall that the instanton $U(x)=\frac{d_{N}}{\left(1+|x|^{2}\right)^{\frac{N-2}{2}}}$, where $d_{N}>0$ is a normalizing constant, satisfies the equation

$$
-\Delta u=|u|^{2^{*}-2} u \text { in } \mathbb{R}^{N} .
$$

Furthermore, we have $\int_{\mathbb{R}^{N}} U^{2^{*}} d x=\int_{\mathbb{R}^{N}}|\nabla U|^{2} d x=S^{\frac{N}{2}}$. We set

$$
U_{\epsilon, y}(x)=\frac{d_{N} \epsilon^{\frac{N-2}{2}}}{\left(\epsilon^{2}+|x-y|^{2}\right)^{\frac{N-2}{2}}}, \quad y \in \mathbb{R}^{N}, \quad \epsilon>0 .
$$

We now observe that

$$
\int_{\Omega} \frac{1}{|x|^{2}} U_{\epsilon, y}(x)^{2} d x=O\left(\epsilon^{2}\right) \quad \text { if } y \neq 0
$$

and

$$
c_{1} \leq \int_{\Omega} \frac{1}{|x|^{2}} U_{\epsilon, 0}(x)^{2} d x \leq c_{2}
$$

for all $\epsilon>0$, where $c_{1}, c_{2}>0$ are constants. We always have

$$
S_{\mu} \leq S_{\infty}
$$

This follows by testing the functional $E_{\mu, Q}$ with $U_{\epsilon, y}$ centered at a point $y$ where either $Q_{m}$ or $Q_{M}$ is achieved. However, if $Q_{M}$ is is achieved only at 0 we cannot 
use directly $U_{\epsilon, 0}$ since by (8) $\int_{\Omega} \frac{1}{|x|^{2}} U_{\epsilon, 0} d x$ is bounded away from 0 for small $\epsilon>0$. In this case we choose a sequence $y_{k} \rightarrow 0$. Testing $E_{\mu, Q}$ with $U_{\epsilon, y_{k}}$ we obtain the estimate $S_{\mu} \leq \frac{S}{Q\left(y_{k}\right)^{\frac{N-2}{N}}}$. Letting $y_{k} \rightarrow 0$ the desired estimate follows. By testing the functional $E_{\mu, Q}$ with $u=1$ we get

$$
S_{\mu} \leq \frac{\mu \int_{\Omega} \frac{1}{|x|^{2}} d x}{\left(\int_{\Omega} Q(x) d x\right)^{\frac{2}{2 *}}} .
$$

Therefore by Proposition 2.2 problem (1) for $\mu<\frac{S_{\infty}\left(\int_{\Omega} Q(x) d x\right)^{\frac{2}{2^{*}}}}{\int_{\Omega} \frac{1}{|x|^{2}} d x}$ has a least energy solution.

\section{Existence and nonexistence of least energy solutions for (1)}

We distinguish two cases: (i) $Q_{M} \leq 2^{\frac{2}{N-2}} Q_{m}$ and (ii) $Q_{M}>2^{\frac{2}{N-2}} Q_{m}$. We denote by $H(y)$ the mean curvature of $\partial \Omega$ at $y \in \partial \Omega$. If $Q=1$ we set $E_{\mu}=E_{\mu, 1}$. It is known that

$$
\begin{aligned}
& E_{\mu}\left(U_{\epsilon, y}\right) \leq \\
& \quad \leq \frac{S}{2^{\frac{2}{N}}}- \begin{cases}A_{N} H(y) \epsilon \log \frac{1}{\epsilon}-a_{N} \mu \epsilon+O(\epsilon)+o(\mu \epsilon), & N=3 \\
A_{N} H(y) \epsilon-a_{N} \mu \epsilon^{2} \log \frac{1}{\epsilon}+O\left(\epsilon^{2} \log \frac{1}{\epsilon}\right)+o\left(\mu \epsilon^{2} \log \frac{1}{\epsilon}\right), & N=4 \\
A_{N} H(y) \epsilon-a_{N} \mu \epsilon^{2}+O\left(\epsilon^{2}\right)+o\left(\mu \epsilon^{2}\right), & N \geq 5\end{cases}
\end{aligned}
$$

where $a_{N}>0$ is a constant depending on $N$. The asymptotic estimation (9) has been established in [3] and [31] for $E_{\mu}$ with the singular term $\frac{u^{2}}{|x|^{2}}$ replaced by $u^{2}$. Since $y \in \partial \Omega$ and $0 \in \Omega$, the proof of (9) is the same as in the nonsingular case.

Theorem 3.1. Let $Q_{M} \leq 2^{\frac{2}{N-2}} Q_{m}$. Suppose that

$$
|Q(x)-Q(y)|=o(|x-y|)
$$

for $x$ near $y$ with $Q(y)=Q_{m}$ and $H(y)>0$. Then for every $\mu>0$ problem (1) has a least energy solution.

Proof. Under our assumptions $S_{\infty}=\frac{S}{2^{\frac{2}{N}} Q_{m^{\frac{N-2}{N}}}}$. Using (9) and (10) we get

$$
E_{\mu, Q}\left(U_{\epsilon, y}\right)<\frac{S}{2^{\frac{2}{N}} Q_{m^{\frac{N-2}{N}}}}
$$

for $\epsilon>0$ sufficiently small. The result follows from Proposition 2.2. 
We now consider the case $Q_{M}>2^{\frac{2}{N-2}} Q_{m}$. We recall the existence result from [15] for the Neumann problem without the singular term

$$
\begin{cases}-\Delta u+\lambda u & =Q(x)|u|^{2^{*}-2} u \text { in } \Omega \\ \frac{\partial}{\partial \nu} u(x) & =0 \text { on } \partial \Omega .\end{cases}
$$

Theorem 3.2. Suppose that $Q_{M}>2^{\frac{2}{N-2}} Q_{m}$. Then there exists a constant $\bar{\Lambda}>0$ such that for $0<\lambda \leq \bar{\Lambda}$ problem (11) has a least energy solution and no least energy solution for $\lambda>\bar{\Lambda}$ and moreover

$$
\frac{S}{Q_{M}^{\frac{N-2}{N}}}=\inf _{u \in H^{1}(\Omega)-\{0\}} \frac{\int_{\Omega}\left(|\nabla u|^{2}+\lambda u^{2}\right) d x}{\left(\int_{\Omega} Q(x)|u|^{2^{*}} d x\right)^{\frac{2}{2^{*}}}}
$$

for $\lambda \geq \bar{\Lambda}$.

Theorem 3.3. Suppose that $Q_{M}>2^{\frac{2}{N-2}} Q_{m}$. Then there exists a constant $\Lambda_{1}>0$ such that for $0<\mu<\Lambda_{1}$ problem (1) has a least energy solution and no least energy solution for $\mu>\Lambda_{1}$ and moreover $S_{\mu}=\frac{S}{Q_{M}^{\frac{N-2}{N}}}$ for $\mu \geq \Lambda_{1}$.

Proof. Let $r=\inf _{x \in \bar{\Omega}-\{0\}} \frac{1}{|x|^{2}}>0$. Then for every $u \in H^{1}(\Omega)$ we have

$$
\int_{\Omega}\left(|\nabla u|^{2}+\mu r u^{2}\right) d x \leq \int_{\Omega}\left(|\nabla u|^{2}+\frac{\mu}{|x|^{2}} u^{2}\right) d x .
$$

Hence

$$
\inf \left\{\int_{\Omega}\left(|\nabla u|^{2}+\mu r u^{2}\right) d x ; u \in H^{1}(\Omega) ; \int_{\Omega} Q(x)|u|^{2^{*}} d x=1\right\} \leq S_{\mu}
$$

and the result follows from Theorem 3.2.

Theorems 3.1 and 3.3 remain true for the problem

$$
\begin{cases}-\Delta u+\frac{\mu}{|x|^{2}} u+\lambda u & =Q(x)|u|^{2^{*}-2} u \text { in } \Omega, \\ \frac{\partial}{\partial \nu} u(x) & =0 \text { on } \partial \Omega,\end{cases}
$$

where $\mu>0$ and $\lambda>0$. We set

$$
S_{\mu, \lambda}=\inf \left\{\int_{\Omega}\left(|\nabla u|^{2}+\frac{\mu}{|x|^{2}}+\lambda u^{2}\right) d x ; u \in H^{1}(\Omega), \int_{\Omega} Q(x)|u|^{2^{*}} d x=1\right\} .
$$

Theorem 3.4. Let $Q_{M} \leq 2^{\frac{2}{N-2}} Q_{m}$ and suppose that (10) holds. Then problem (12) has a least energy solution for every $\mu>0$ and $\lambda>0$. 
Theorem 3.5. Let $Q_{M}>2^{\frac{2}{N-2}}$. Then there exists $\Lambda>0$ such that for $\lambda+\mu r<\Lambda$ problem (12) has a least energy solution and no least energy solution for $\lambda+\mu r>\Lambda$. Moreover, $S_{\mu, \lambda}=\frac{S}{Q_{M}^{\frac{N-2}{N}}}$ for $\lambda+\mu r \geq \Lambda$.

If $Q_{M} \leq 2^{\frac{2}{N-2}} Q_{m}$ and $Q_{m}$ is achieved only at points of $\partial \Omega$ with the negative mean curvature then least energy solutions do not exist for large $\mu>0$. This follow from the following result [14]:

Theorem 3.6. Let $N \geq 5$ and $Q_{M} \leq 2^{\frac{2}{N-2}} Q_{m}$. Suppose that (10) holds and moreover

$$
\{x \in \partial \Omega ; H(x)<0\} \neq \emptyset \quad \text { and } \quad\left\{x \in \partial \Omega ; Q(x)=Q_{m}\right\} \subset\{x \in \partial \Omega ; H(x)<0\} .
$$

Then there exists $\tilde{\lambda}>0$ such that for $0<\lambda \leq \tilde{\lambda}$ problem (11) has a least energy solution and no least energy solution for $\lambda>\tilde{\lambda}$ and

$$
\frac{S}{2^{\frac{2}{N}} Q_{m^{N}}^{\frac{N-2}{N}}}=\inf _{H^{1}\left(\Omega_{)}-\{0\}\right.} \frac{\int_{\Omega}\left(|\nabla u|^{2}+\lambda u^{2}\right) d x}{\left(\int_{\Omega} Q(x)|u|^{2^{*}} d x\right)^{\frac{2}{2^{*}}}}
$$

for $\lambda \geq \tilde{\lambda}$

Theorem 3.7. Suppose that the assumptions of Theorem 3.6 hold. Then there exists a constant $\tilde{\Lambda}>0$ such that for $0<\mu<\tilde{\Lambda}$ problem (1) has a least energy solution and no least energy solution for $\mu>\tilde{\Lambda}$ and moreover $S_{\mu}=\frac{S}{2^{\frac{2}{N}} Q_{m^{\frac{N-2}{N}}}}$ for $\mu \geq \tilde{\Lambda}$.

We now examine $S_{\mu, \lambda}$ as a function of $\lambda$ for a fixed $\mu>0$. It is clear that $S_{\mu, \lambda}$ is continuous and non decreasing. It is also bounded from above by $S_{\infty}$. Testing $S_{\mu, \lambda}$ with $u=1$ on $\Omega$, we see that

$$
S_{\mu, \lambda} \leq \frac{\mu \int_{\Omega} \frac{d x}{|x|^{2}}+\lambda|\Omega|}{\left(\int_{\Omega} Q(x) d x\right)^{\frac{2}{2^{*}}}}
$$

Hence $\lim _{\lambda \rightarrow-\infty} S_{\mu, \lambda}=-\infty$. We show, below in Proposition 3.8, that $S_{\mu, \lambda}$ admits a minimizer for every $\lambda \in \mathbb{R}$ with $S_{\mu, \lambda} \leq 0$. However, these minimizers do not satisfy (12).

Proposition 3.8. (i) If $S_{\mu, \lambda}<0$ for some $\mu>0$ and $\lambda \in \mathbb{R}$, then there exists a minimizer $u$ for $S_{\mu, \lambda}$ which after rescaling $\left|S_{\mu, \lambda}\right|^{\frac{1}{2^{*}-2}} u$ satisfies

$$
\begin{cases}-\Delta u+\frac{\mu}{|x|^{2}} u+\lambda u & =-Q(x)|u|^{2^{*}-2} u \text { in } \Omega \\ \frac{\partial}{\partial \nu} u(x) & =0 \text { on } \partial \Omega\end{cases}
$$


(ii) There exists a unique $\lambda_{\circ} \leq-\mu$ such that $S_{\mu, \lambda_{\circ}}=0$, where $r=\min _{x \in \bar{\Omega}-\{0\}} \frac{1}{|x|^{2}}$. Moreover, $-\lambda_{\circ}$ is an eigenvalue of the following problem

$$
\begin{cases}-\Delta u+\frac{\mu}{|x|^{2}} u & =\sigma u \text { in } \Omega \\ \frac{\partial}{\partial \nu} u(x) & =0 \text { on } \partial \Omega .\end{cases}
$$

Proof. Suppose that $S_{\mu, \lambda}<0$ for some $\lambda \in \mathbb{R}$. Let $\left\{u_{m}\right\}$ be a minimizing sequence for $S_{\mu, \lambda}$, that is,

$$
\int_{\Omega}\left(\left|\nabla u_{m}\right|^{2}+\frac{\mu}{|x|^{2}} u_{m}^{2}+\lambda u_{m}^{2}\right) d x \rightarrow S_{\mu, \lambda} \text { and } \int_{\Omega} Q(x)\left|u_{m}\right|^{2^{*}} d x=1 .
$$

Since $\left\{u_{m}\right\}$ is bounded in $H^{1}(\Omega)$ we may assume that $u_{m} \rightarrow u$ in $H^{1}(\Omega)$ and $L^{2^{*}}(\Omega)$ and $u_{m} \rightarrow u$ in $L^{2}(\Omega)$. It follows from (15) that $u \neq 0$. We claim that $\int_{\Omega} Q(x)|u|^{2^{*}} d x=1$. In the contrary case there exists $t>1$ such that

$$
t^{2^{*}} \int_{\Omega} Q(x)|u|^{2^{*}} d x=1
$$

Then by the lower semicontinuity of a norm with respect to a weak convergence, we have

$$
S_{\mu, \lambda} \leq t^{2} \int_{\Omega}\left(|\nabla u|^{2}+\frac{\mu}{|x|^{2}} u^{2}+\lambda u^{2}\right) d x \leq t^{2} S_{\mu, \lambda} .
$$

Since $S_{\mu, \lambda}<0$, we must have $t^{2} \leq 1$, which is impossible. Thus $\int_{\Omega} Q(x)|u|^{2^{*}} d x=1$ and $u$ is a minimizer. Letting $v=\left|S_{\mu, \lambda}\right|^{\frac{1}{2^{*}-2}} u$, we verify that $v$ is a solution of problem (13). If $S_{\mu, \lambda_{\circ}}=0$ for some $\lambda_{\circ}=\lambda_{\circ}(\mu)<0$, then the limit $u$ of a minimizing sequence $\left\{u_{m}\right\}$ must be nonzero. Indeed, if $u=0$ on $\Omega$, then (15) yields $u_{m} \rightarrow 0$ in $H^{1}(\Omega)$ which is impossible. If $\int_{\Omega} Q(x)|u|^{2^{*}} d x<1$, then a suitable multiple $t u$ for some $t>1$ is a minimizer. By the continuity of $S_{\mu, \lambda}$ we can find $\delta>0$ such that $S_{\mu, \lambda}<S_{\infty}$ for every $\lambda<\lambda_{\circ}+\delta$. Since $S_{\mu, \lambda}$ is attained for each $\lambda<\lambda_{\circ}+\delta, S_{\mu, \lambda}$ is strictly increasing on this interval. Therefore $S_{\mu, \lambda}$ vanishes only at $\lambda_{\circ}$. On the other hand considering the Rayleigh quotient for the first eigenvalue we get

$$
\inf _{u \in H^{1}(\Omega)-\{0\}} \frac{\int_{\Omega}\left(|\nabla u|^{2}+\frac{\mu}{|x|^{2}} u^{2}\right) d x}{\int_{\Omega} u^{2} d x} \geq \mu r
$$

Hence $\lambda_{\circ} \leq-\mu r$.

\section{Problem (12) with $\boldsymbol{\mu}<0$}

It is convenient to write problem (12) with $\mu<0$ in the following way

$$
\begin{cases}-\Delta u-\frac{\mu}{|x|^{2}} u+\lambda u & =Q(x)|u|^{2^{*}-2} u \text { in } \Omega, \\ \frac{\partial}{\partial \nu} u(x) & =0 \text { on } \partial \Omega\end{cases}
$$


where $\mu>0$ and $\lambda \in \mathbb{R}$. To find solutions of (16) we consider the constrained minimization problem

$$
S_{-\mu, \lambda}=\inf _{u \in H^{1}(\Omega)-\{0\}} \frac{\int_{\Omega}\left(|\nabla u|^{2}-\frac{\mu}{|x|^{2}} u^{2}+\lambda u^{2}\right) d x}{\left(\int_{\Omega} Q(x)|u|^{2^{*}} d x\right)^{\frac{2}{2^{*}}}} .
$$

First we consider the case where $S_{-\mu, \lambda}>0$. To examine the concentration phenomena of minimizing sequences we need the following quantity

$$
S_{-\mu}=\inf _{D^{1,2}\left(\mathbb{R}^{N}\right)-\{0\}} \frac{\int_{\mathbb{R}^{N}}\left(|\nabla u|^{2}-\frac{\mu}{|x|^{2}} u^{2}\right) d x}{\left(\int_{\mathbb{R}^{N}}|u|^{2^{*}} d x\right)^{\frac{2}{2^{*}}}} .
$$

It is known [18] that if $0<\mu<\bar{\mu}=\frac{(N-2)^{2}}{4}=\frac{1}{c_{N}}$, then

$$
S_{-\mu}=\inf _{u \in H_{\circ}^{1}(\Omega)-\{0\}} \frac{\int_{\Omega}\left(|\nabla u|^{2}-\mu \frac{u^{2}}{|x|^{2}}\right) d x}{\left(\int_{\Omega}|u|^{2^{*}} d x\right)^{\frac{2}{2^{*}}}},
$$

which means that the inf over $H_{\circ}^{1}(\Omega)$ is independent of $\Omega$. If $\Omega=\mathbb{R}^{N}$ the constant $S_{-\mu}$ is attained by a family of functions (see [29])

$$
U_{\epsilon}^{\mu}(x)=\frac{k_{N} \epsilon^{\sqrt{\bar{\mu}-\mu}}}{\left(\epsilon^{\frac{\gamma-\gamma^{\prime}}{\sqrt{\bar{\mu}}}}|x|^{\frac{\gamma^{\prime}}{\sqrt{\bar{\mu}}}}+|x|^{\frac{\gamma}{\sqrt{\mu}}}\right)^{\sqrt{\bar{\mu}}}}, \quad \epsilon>0,
$$

where $k_{N}>0$ is a normalizing constant and $\gamma=\sqrt{\bar{\mu}}+\sqrt{\bar{\mu}-\mu}$ and $\gamma^{\prime}=\sqrt{\bar{\mu}}-\sqrt{\bar{\mu}-\mu}$. We also have

$$
\int_{\mathbb{R}^{N}}\left|\nabla U_{\epsilon}^{\mu}(x)\right|^{2} d x-\mu \int_{\mathbb{R}^{N}} \frac{\left(U_{\epsilon}^{\mu}(x)\right)^{2}}{|x|^{2}} d x=\int_{\mathbb{R}^{N}}\left(U_{\epsilon}(x)^{\mu}\right)^{2^{*}} d x=S_{-\mu}^{\frac{N}{2}} .
$$

We obviously have $S_{-\mu}<S$ for $\mu<\bar{\mu}$ and $\lim _{\mu \rightarrow 0} S_{-\mu}=S$. On the other hand the constant in (17) is not attained if $\Omega$ is a bounded star-shaped domain containing the origin (see [21]).

Let $S_{-\mu, \lambda}>0$. Suppose that $\left\{u_{m}\right\}$ is a minimizing sequence for $S_{-\mu, \lambda}$. Since $\left\{u_{m}\right\}$ is bounded in $H^{1}(\Omega)$, we may assume that $u_{m} \rightarrow u$ in $H^{1}(\Omega), L^{2^{*}}(\Omega)$ and $u_{m} \rightarrow u$ in $L^{2}(\Omega)$. It follows from the concentration-compactness principle that

$$
\left|u_{n}\right|^{2^{*}} \stackrel{*}{\rightarrow}|u|^{2^{*}}+\sum_{j \in J} \nu_{j} \delta_{x_{j}}+\nu_{\circ} \delta_{\circ}
$$

and

$$
\left|\nabla u_{m}\right|^{2}-\mu \frac{u_{m}^{2}}{|x|^{2}} \stackrel{*}{\rightarrow}|\nabla u|^{2}-\mu \frac{u^{2}}{|x|^{2}}+\sum_{j \in J} \mu_{j} \delta_{x_{j}}+\mu_{\circ} \delta_{\circ},
$$


where $\mu_{j}, \mu_{\circ}, \nu_{j}$ and $\nu_{\circ}$ are positive constant and $J$ is at most countable set. The sequence $\left\{\frac{u_{m}^{2}}{|x|^{2}}\right\}$ can only concentrate at 0 . Hence $\mu_{\circ} \delta_{\circ}$ is a joint effect of the concentration of $\left\{\left|\nabla u_{m}\right|^{2}\right\}$ and $\left\{\frac{u_{m}^{2}}{|x|^{2}}\right\}$ at 0 . Moreover, we have

$$
\begin{gathered}
S \nu_{j}^{\frac{2}{2^{*}}} \leq \mu_{j} \text { if } x_{j} \neq 0 \text { and } x_{j} \in \Omega, \\
\frac{S}{2^{\frac{2}{N}}} \nu_{j}^{\frac{2}{2^{*}}} \leq \mu_{j} \text { if } x_{j} \in \partial \Omega
\end{gathered}
$$

and

$$
\bar{S}_{\infty}=\min \left(\frac{S}{2^{\frac{2}{N}} Q_{m}^{\frac{N-2}{N}}}, \frac{S}{Q_{M}^{\frac{N-2}{N}}}, \frac{S_{-\mu}}{Q(0)^{\frac{N-2}{N}}}\right) .
$$

Proposition 4.1. Suppose that

$$
0<S_{-\mu, \lambda}<\bar{S}_{\infty}
$$

for some $0<\mu<\bar{\mu}$ and $\lambda \in \mathbb{R}$. Then problem (16) has a least energy solution.

The proof is similar to that of Proposition 2.2 and is omitted.

To apply Proposition 4.1 we must ensure the existence of $\lambda$ and $\mu \in(0, \bar{\mu})$ such that $0<S_{-\mu, \lambda}<\bar{S}_{\infty}$. It follows from Lemma 2.1 that for every $\delta>0$ there exists a constant $C(\delta)>0$ such that

$$
\begin{aligned}
\left(1-\left(c_{N}+\delta\right) \mu\right) \int_{\Omega}|\nabla u|^{2} d x+(\lambda-C(\delta) \mu) & \int_{\Omega} u^{2} d x \leq \\
& \leq \int_{\Omega}\left(|\nabla u|^{2}-\frac{\mu}{|x|^{2}} u^{2}+\lambda u^{2}\right) d x
\end{aligned}
$$

for every $u \in H^{1}(\Omega)$. Choosing $\delta>0$ so that $\left(1-\left(c_{N}+\delta\right) \mu\right)>0$ and then taking $\lambda>$ $C(\delta)$ we can guarantee $S_{-\mu, \lambda}$ to be positive. Testing $S_{-\mu, \lambda}$ with constant functions we deduce that $\lim _{\lambda \rightarrow-\infty} S_{-\mu, \lambda}=-\infty$ for each $\mu \in\left(0, \frac{1}{c_{N}}\right)$. We are now in a position to formulate the existence results for problem (16).

Theorem 4.2. (i) Suppose that $\bar{S}_{\infty}=\frac{S}{2^{\frac{2}{N}} Q_{m^{\frac{N-2}{N}}}}$. If (10) holds, then for every $0<\mu<\bar{\mu}$, there exists $\bar{\lambda}=\bar{\lambda}(\mu)>0$ such that for every $0<\mu<\bar{\mu}$ and $\bar{\lambda}<\lambda$ problem (16) has a least energy solution.

(ii) Suppose that $\bar{S}_{\infty}=\frac{S}{Q_{M}^{\frac{N-2}{N}}}$. Then there exists $\Lambda>0$ such that for every $0<$ $\lambda_{\circ}<\Lambda$ there exists $0<\mu_{\circ}<\bar{\mu}$ such that for $0<\mu<\mu_{\circ}$ and $\lambda_{\circ}<\lambda<\Lambda$ problem (16) has a least energy solution. 
(iii) Suppose that $\bar{S}_{\infty}=\frac{S_{-\mu}}{Q(0)^{\frac{N-2}{N}}}$ Then there exists $\lambda^{*}>0$ such that for every $0<\lambda_{*}<\lambda^{*}$ there exists $0<\mu_{\circ}<\bar{\mu}$ such that for every $\lambda_{*}<\lambda<\lambda^{*}$ and $0<\mu<\mu_{\circ}$ problem (16) has a least energy solution.

Proof. (i) The estimate (18) shows that for every $0<\mu<\bar{\mu}$ there exists $\bar{\lambda}(\mu)$ such that $S_{-\mu, \lambda}>0$ for $\lambda>\bar{\lambda}$. We now apply the asymptotic estimate (9) to verify that

$$
S_{-\mu, \lambda}<\frac{S}{2^{\frac{2}{N}} Q_{m^{\frac{N-2}{N}}}}
$$

for $\lambda>\bar{\lambda}$.

(ii) First, we observe that

$$
S_{-\mu, \lambda} \leq S_{0, \lambda}
$$

for every $0<\mu<\bar{\mu}$ and $\lambda \in \mathbb{R}$. According to Theorem 3.2 there exists $\Lambda>0$ such that $S_{0, \lambda}<\frac{S}{Q_{M}^{\frac{N}{N}}}$ for every $0<\lambda<\Lambda$ and $S_{0, \lambda}=\frac{S}{Q_{M}^{\frac{N}{N}}}$ for $\lambda \geq \Lambda$. Let $\lambda_{\circ}<\Lambda$ be given. Then using (18) we may choose $0<\mu_{\circ}<\bar{\mu}$ such that $S_{-\mu, \lambda}>0$ for every $0<\mu<\mu_{\circ}$ and $\lambda_{\circ}<\lambda<\Lambda$. The result follows from Proposition 4.1.

(iii) In this case using (19) and the fact that $\lim _{\lambda \rightarrow 0} S_{0, \lambda}=0$ we can find $\lambda^{*}>0$ such that

$$
S_{-\mu, \lambda}<\frac{S_{-\mu}}{Q(0)^{\frac{N-2}{N}}}
$$

for every $\lambda<\lambda^{*}$. It then follows from (18) that given $\lambda_{*}<\lambda^{*}$ we can choose $0<\mu_{\circ} \leq \bar{\mu}$ so that $0<S_{-\mu, \lambda}$ for $0<\mu \leq \mu_{\circ}$ and $\lambda_{*} \leq \lambda \leq \lambda^{*}$ and the result follows.

We now establish a result for problem (16) which analogous to Proposition 3.8.

Proposition 4.3. (i) Let $S_{-\mu, \lambda}<0$ for some $\lambda \in \mathbb{R}$. Then there exists a minimizer $u$ and $\left|S_{-\mu, \lambda}\right|^{\frac{1}{2^{*}-2}} u$ is a solution of problem

$$
\begin{cases}-\Delta u-\frac{\mu}{|x|^{2}} u+\lambda u & =-Q(x)|u|^{2^{*}-2} u \text { in } \Omega \\ \frac{\partial}{\partial \nu} u(x) & =0 \text { on } \partial \Omega\end{cases}
$$

(ii) There exists a $\lambda_{\circ} \geq \mu r$ such that $S_{-\mu, \lambda_{\circ}}=0$ and $-\lambda_{\circ}$ is an eigenvalue of the problem

$$
\begin{cases}-\Delta u-\frac{\mu}{|x|^{2}} u & =\sigma u \text { in } \Omega \\ \frac{\partial}{\partial \nu} u(x) & =0 \text { on } \partial \Omega .\end{cases}
$$

Proof. (i) Let $\left\{u_{m}\right\}$ be a minimizing sequence for $S_{-\mu, \lambda}<0$, that is,

$$
\int_{\Omega}\left(\left|\nabla u_{m}\right|^{2}-\frac{\mu}{|x|^{2}} u_{m}^{2}+\lambda u_{m}^{2}\right) d x=S_{-\mu, \lambda}+o(1) \text { and } \int_{\Omega} Q(x)\left|u_{m}\right|^{2^{*}} d x=1
$$


for every $m$. We may assume that $u_{m} \rightarrow u$ in $H^{1}(\Omega), L^{2^{*}}(\Omega)$ and $u_{m} \rightarrow u$ on $L^{2}(\Omega)$. By Lemma 2.1 for every $\delta>0$, with $\mu\left(c_{N}+\delta\right)<1$, there exists $C(\delta)>0$ such that

$$
\left(1-\left(c_{N}+\delta\right) \mu\right) \int_{\Omega}\left|\nabla u_{m}\right|^{2} d x+(\lambda-C(\delta) \mu) \int_{\Omega} u_{m}^{2} d x \leq S_{-\mu, \lambda}+o(1) .
$$

This yields $u \neq 0$. We now show that $\int_{\Omega} Q(x)|u|^{2^{*}} d x=1$. In the contrary case we have $\int_{\Omega} Q(x)|t u|^{2^{*}} d x=1$ for some $t>1$. On the other hand by the Lieb-Brézis lemma [10], letting $v_{m}=u_{m}-u$, we get

$$
\begin{aligned}
\int_{\Omega}|\nabla u|^{2} d x & +\int_{\Omega}\left|\nabla v_{m}\right|^{2} d x-\mu \int_{\Omega} \frac{u^{2}}{|x|^{2}} d x-\mu \int_{\Omega} \frac{v_{m}^{2}}{|x|^{2}} d x \\
& +\lambda \int_{\Omega} u^{2} d x+\lambda \int_{\Omega} v_{m}^{2} d x=S_{-\mu, \lambda}+o(1) .
\end{aligned}
$$

Let $0<\delta$ and $\left(c_{N}+\delta\right) \mu<1$. Applying Lemma 2.1 we get

$$
\begin{gathered}
\left(1-\left(c_{N}+\delta\right) \mu\right) \int_{\Omega}\left|\nabla v_{m}\right|^{2} d x+\int_{\Omega}|\nabla u|^{2} d x-\mu \int_{\Omega} \frac{u^{2}}{|x|^{2}} d x \\
+(\lambda-\mu C(\delta)) \int_{\Omega} v_{m}^{2} d x+\lambda \int_{\Omega} u^{2} d x \leq S_{-\mu, \lambda}+o(1) .
\end{gathered}
$$

Since $v_{m} \rightarrow 0$ in $L^{2}(\Omega)$, we deduce from the above inequality that

$$
\int_{\Omega}\left(|\nabla u|^{2}-\mu \frac{u^{2}}{|x|^{2}}+\lambda u^{2}\right) d x \leq S_{-\mu, \lambda}
$$

From this we derive that

$$
S_{-\mu, \lambda} \leq t^{2} \int_{\Omega}\left(|\nabla u|^{2}-\mu \frac{u^{2}}{|x|^{2}}+\lambda u^{2}\right) d x \leq S_{-\mu, \lambda} t^{2} .
$$

Since $S_{-\mu, \lambda}<0$, we see that $t^{2} \leq 1$ which is impossible. Therefore $u$ is a minimizer.

(ii) In a similar manner we show that if $S_{-\mu, \lambda_{\circ}}=0$ for some $\lambda_{\circ}$, then there exists a minimizer $u$ satisfying (21). By the continuity of $S_{-\mu, \lambda}$ there exists $\delta>0$ such that $S_{-\mu, \lambda}<\bar{S}_{\infty}$ for $\lambda \in\left(-\infty, \lambda_{\circ}+\delta\right)$. Since for every $\lambda \in\left(-\infty, \lambda_{\circ}+\delta\right) S_{-\mu, \lambda}$ is achieved, $S_{-\mu, \lambda}$ is strictly increasing and $\lambda_{\circ}$ is unique.

\section{Eigenvalue problems}

We consider two eigenvalue problems (14) and (21). We begin by proving the existence of the first eigenvalues denoted by $\lambda_{1}^{\mu}$ and $\lambda_{1}^{-\mu}$, respectively.

Proposition 5.1. (i) For every $\mu>0$ there exists the first (smallest) eigenvalue $\lambda_{1}^{\mu}$ of problem (14) which satisfies $\lambda_{1}^{\mu} \geq \mu$. 
(ii) For every $0<\mu<\frac{1}{c_{N}}$ there exists the first (smallest) eigenvalue $\lambda_{1}^{-\mu}$ of problem (21) which satisfies $\lambda_{1}^{-\mu} \leq-\mu r$.

Proof. (ii) We define $\lambda_{1}^{-\mu}$ by

$$
\lambda_{1}^{-\mu}=\inf _{u \in H^{1}(\Omega)-\{0\}} \frac{\int_{\Omega}\left(|\nabla u|^{2}-\mu \frac{u^{2}}{|x|^{2}}\right) d x}{\int_{\Omega} u^{2} d x} .
$$

Using Lemma 2.1 we verify that $\lambda_{1}^{-\mu}>-\infty$. It is clear that $\lambda_{1}^{-\mu} \leq-\mu r$. Let $\left\{u_{m}\right\}$ be a minimizing sequence for $\lambda_{1}^{-\mu}$. Then

$$
\int_{\Omega}\left(\left|\nabla u_{m}\right|^{2}-\frac{\mu}{|x|^{2}} u_{m}^{2}\right) d x=\lambda_{1}^{-\mu}+o(1) \text { and } \int_{\Omega}\left|u_{m}\right|^{2} d x=1 .
$$

With the aid of Lemma 2.1 we show that $\left\{u_{m}\right\}$ is bounded in $H^{1}(\Omega)$. We may assume that $u_{m} \rightarrow u$ in $H^{1}(\Omega)$ and $u_{m} \rightarrow u$ in $L^{2}(\Omega)$. Letting $v_{m}=u_{m}-u$ we have

$$
\begin{gathered}
\int_{\Omega}\left|\nabla u_{m}\right|^{2} d x=\int_{\Omega}\left|\nabla v_{m}\right|^{2} d x+\int_{\Omega}|\nabla u|^{2} d x+o(1), \\
\int_{\Omega} \frac{u_{m}^{2}}{|x|^{2}} d x=\int_{\Omega} \frac{v_{m}^{2}}{|x|^{2}} d x+\int_{\Omega} \frac{u^{2}}{|x|^{2}} d x+o(1)
\end{gathered}
$$

and

$$
\int_{\Omega} u_{m}^{2} d x=1+o(1)
$$

Substituting these relations into (22) we get

$$
\int_{\Omega}\left|\nabla v_{m}\right|^{2} d x+\int_{\Omega}|\nabla u|^{2} d x-\mu \int_{\Omega} \frac{v_{m}^{2}}{|x|^{2}} d x-\mu \int_{\Omega} \frac{u^{2}}{|x|^{2}} d x=\lambda_{1}^{-\mu}+o(1) .
$$

We fix $\delta>0$ so that $\mu+\delta<\frac{1}{c_{N}}$. Then $\lambda_{1}^{-(\mu+\delta)}>-\infty$. Since

$$
\lambda_{1}^{-(\mu+\delta)} \int_{\Omega} v_{m}^{2} d x \leq \int_{\Omega}\left|\nabla v_{m}\right|^{2} d x-(\mu+\delta) \int_{\Omega} \frac{v_{m}^{2}}{|x|^{2}} d x,
$$

we get from (23) that

$$
\lambda_{1}^{-(\mu+\delta)} \int_{\Omega} v_{m}^{2} d x+\delta \int_{\Omega} \frac{v_{m}^{2}}{|x|^{2}} d x+\int_{\Omega}|\nabla u|^{2} d x-\mu \int_{\Omega} \frac{u^{2}}{|x|^{2}} d x \leq \lambda_{1}^{-\mu}+o(1) .
$$

From this we deduce that $\delta \int_{\Omega} \frac{v_{m}^{2}}{|x|^{2}} d x=o(1)$, so $\int_{\Omega} \frac{u_{m}^{2}}{|x|^{2}} d x \rightarrow \int_{\Omega} \frac{u^{2}}{|x|^{2}} d x$. Therefore by (23) we have

$$
\int_{\Omega}\left(|\nabla u|^{2}-\mu \frac{u^{2}}{|x|^{2}}\right) d x \leq \lambda_{1}^{-\mu}
$$

Since $\int_{\Omega} u^{2} d x=1, u$ is a minimizer for $\lambda_{1}^{-\mu}$.

The proof of (i) is similar and is omitted. 
The proof of (ii) strongly relies on the fact that $0<\mu<\frac{1}{c_{N}}$. We were unable to show whether $\lambda_{1}^{-\mu}$ with $\mu=\frac{1}{c_{N}}$ is attained or not. It is known that the constant $\lambda_{1}^{-\mu}$ defined on $H_{\circ}^{1}(\Omega)-\{0\}$ is not attained for $\mu=\frac{1}{c_{N}}$ (see [6], [30]). Also, in the case of $H_{\circ}^{1}(\Omega), \lambda_{1}^{-\mu}$ is positive. Since $H^{1}(\Omega)$ contains constant functions, $\lambda_{1}^{-\mu}$ in our case is negative.

By Lemma $2.1-\Delta-\frac{\mu}{|x|^{2}}+C$ with the Neumann boundary conditions, $0<\mu<\frac{1}{c_{N}}$ and $C>0$ sufficiently large is a positive definite and self-adjoint operator. Therefore its spectrum $\sigma_{-\mu}$ is discrete and consists of an increasing sequence of eigenvalues $\left\{\lambda_{j}^{-\mu, C}\right\}$ converging to infinity as $j \rightarrow \infty$. Eigenvalues $\left\{\lambda_{j}^{-\mu}\right\}$ of the operator $-\Delta-\frac{\mu}{|x|^{2}}$ are given by $\lambda_{j}^{-\mu}=\lambda_{j}^{-\mu, C}-C$. Eigenfunctions of $-\Delta-\frac{\mu}{|x|^{2}}$ can be characterized by usual Rayleigh quotients. In particular, if $\phi$ is an eigenfunction corresponding to the smallest eigenvalue $\lambda_{1}^{-\mu}$, then $|\phi|$ is also an eigenfunction of $\lambda_{1}^{-\mu}$. Consequently we may assume that $\phi \geq 0$ on $\Omega$. Applying Theorem 8.19 (the strong maximum principle for weak solutions) in [22] we can choose $\phi>0$ a. e. on $\Omega$. It is easy to show that the eigenvalue $\lambda_{1}^{-\mu}$ is simple.

Similarly, the spectrum $\sigma_{\mu}$ of $-\Delta+\frac{\mu}{|x|^{2}}$ is also discrete, each eigenvalue $\lambda_{k}^{\mu}$ and has a finite multiplicity. The smallest eigenvalue $\lambda_{1}^{\mu}$ is simple. Moreover $\lambda_{k}^{\mu} \rightarrow \infty$ as $k \rightarrow \infty$.

\section{Topological linking}

A min-max principle based on a topological linking will be used to investigate the existence of solutions of problems (1) and (16) in the cases where a parameter $\lambda$ interferes with the spectrum $\sigma_{\mu}$ and $\sigma_{-\mu}$. We rewrite both problems in the following way

$$
\begin{cases}-\Delta u+\frac{\mu}{|x|^{2}} u & =\lambda u+Q(x)|u|^{2^{*}-2} u \text { in } \Omega \\ \frac{\partial}{\partial \nu} u(x) & =0 \text { on } \partial \Omega .\end{cases}
$$

and

$$
\begin{cases}-\Delta u-\frac{\mu}{|x|^{2}} u & =\lambda u+Q(x)|u|^{2^{*}-2} u \text { in } \Omega \\ \frac{\partial}{\partial \nu} u(x) & =0 \text { on } \partial \Omega\end{cases}
$$

where $\mu>0$ for problem (24) and $0<\mu<\frac{1}{c_{N}}=\bar{\mu}$ for problem (25). The range for a parameter $\lambda$ will be given later. Solutions of problems (24) and (25) will be obtained as critical points of the variational functionals

$$
I_{\mu, \lambda}(u)=\frac{1}{2} \int_{\Omega}\left(|\nabla u|^{2}+\mu \frac{u^{2}}{|x|^{2}}-\lambda u^{2}\right) d x-\frac{1}{2^{*}} \int_{\Omega} Q(x)|u|^{2^{*}} d x
$$

and

$$
I_{-\mu, \lambda}(u)=\frac{1}{2} \int_{\Omega}\left(|\nabla u|^{2}-\mu \frac{u^{2}}{|x|^{2}}-\lambda u^{2}\right) d x-\frac{1}{2^{*}} \int_{\Omega} Q(x)|u|^{2^{*}} d x .
$$


Lemma 6.1. For every $c \in \mathbb{R},(P S)_{c}$ sequences for both functionals $I_{-\mu, \lambda}$ and $I_{\mu, \lambda}$ are bounded in $H^{1}(\Omega)$.

Proof. First, we consider the functional $I_{-\mu, \lambda}$. We assume that $0<\mu<\frac{1}{c_{N}}$. Let $\left\{u_{m}\right\}$ be a $(P S)_{c}$ sequence. Arguing by contradiction assume that $\left\|u_{m}\right\| \rightarrow \infty$. We set $v_{m}=\frac{u_{m}}{\left\|u_{m}\right\|}$. Since $\left\{u_{m}\right\}$ is bounded in $H^{1}(\Omega)$ we may assume that $v_{m} \rightarrow v$ in $H^{1}(\Omega)$ and $v_{m} \rightarrow v$ in $L^{p}(\Omega)$ for every $2 \leq p<2^{*}$. Then

$$
\frac{1}{\left\|u_{m}\right\|^{2^{*}-2}} \int_{\Omega}\left(\nabla v_{m} \nabla \phi-\mu \frac{v_{m} \phi}{|x|^{2}}-\lambda v_{m} \phi\right) d x=\int_{\Omega} Q(x)\left|v_{m}\right|^{2^{*}-2} v_{m} \phi d x+o(1) .
$$

We deduce from this that

$$
\int_{\Omega} Q(x)|v|^{2^{*}-2} v \phi d x=0
$$

for every $\phi \in H^{1}(\Omega)$. This yields $v=0$ a.e. on $\Omega$. Since $\left\{u_{m}\right\}$ is a $(P S)_{c}$ sequence, we get

$$
\frac{1}{2} \int_{\Omega}\left(\left|\nabla v_{m}\right|^{2}-\mu \frac{v_{m}^{2}}{|x|^{2}}-\lambda v_{m}^{2}\right) d x-\frac{1}{2^{*}}\left\|u_{m}\right\|^{2^{*}-2} \int_{\Omega} Q(x)\left|v_{m}\right|^{2^{*}} d x \rightarrow 0
$$

and

$$
\int_{\Omega}\left(\left|\nabla v_{m}\right|^{2}-\mu \frac{v_{m}^{2}}{|x|^{2}}-\lambda v_{m}^{2}\right) d x-\left\|u_{m}\right\|^{2^{*}-2} \int_{\Omega} Q(x)\left|v_{m}\right|^{2^{*}} d x \rightarrow 0
$$

as $m \rightarrow \infty$. Since $v_{m} \rightarrow 0$ in $L^{2}(\Omega)$, these two relations yield that

$$
\lim _{m \rightarrow \infty} \int_{\Omega}\left(\left|\nabla v_{m}\right|^{2}-\mu \frac{v_{m}^{2}}{|x|^{2}}\right) d x=0
$$

and

$$
\lim _{m \rightarrow \infty}\left\|u_{m}\right\|^{2^{*}-2} \int_{\Omega} Q(x)\left|v_{m}\right|^{2^{*}} d x=0 .
$$

We now apply Lemma 2.1 with $\delta>0$ chosen so that $\mu\left(c_{N}+\delta\right)<1$. Thus

$$
\int_{\Omega}\left(\left|\nabla v_{m}\right|^{2}-\mu \frac{v_{m}^{2}}{|x|^{2}}\right) d x \geq\left(1-\mu\left(c_{N}+\delta\right)\right) \int_{\Omega}\left|\nabla v_{m}\right|^{2} d x-C(\delta) \mu \int_{\Omega} v_{m}^{2} d x
$$

for some constant $C(\delta)>0$. Since $v_{m} \rightarrow 0$ in $L^{2}(\Omega)$, we deduce from (26) and (27) that $\lim _{m \rightarrow \infty} \int_{\Omega}\left|\nabla v_{m}\right|^{2} d x=0$, which is a contradiction.

We now consider the functional $I_{\mu, \lambda}$ with $\mu>0$. If $\left\{u_{m}\right\}$ is a $(P S)_{c}$ sequence of this functional, then

$$
\frac{1}{N} \int_{\Omega} Q(x)\left|u_{m}\right|^{2^{*}} d x=I_{\mu, \lambda}\left(u_{m}\right)-\frac{1}{2}\left\langle I_{\mu, \lambda}^{\prime}\left(u_{m}\right), u_{m}\right\rangle=c+o\left(\left\|u_{m}\right\|\right)+o(1) .
$$


Hence

$$
\int_{\Omega} Q(x)\left|u_{m}\right|^{2^{*}} d x \leq C_{1}+C_{2}\left\|u_{m}\right\|
$$

and

$$
\int_{\Omega} u_{m}^{2} d x \leq C_{1}+C_{2}\left\|u_{m}\right\|
$$

for some constants $C_{1}, C_{2}>0$ and all $m$. These two estimates combined with the inequality

$$
\begin{aligned}
\frac{1}{2} \int_{\Omega}\left(\left|\nabla u_{m}\right|^{2}+\mu r u_{m}^{2}\right) d x & \leq \frac{1}{2} \int_{\Omega}\left(\left|\nabla u_{m}\right|^{2}+\mu \frac{u_{m}^{2}}{|x|^{2}}\right) d x \\
& =c+\frac{\lambda}{2} \int_{\Omega} u_{m}^{2} d x+\frac{1}{2^{*}} \int_{\Omega} Q(x)\left|u_{m}\right|^{2^{*}} d x+o(1)
\end{aligned}
$$

imply that the sequence $\left\{u_{m}\right\}$ is bounded in $H^{1}(\Omega)$.

To proceed further we set

$$
S_{\infty, h}=\min \left(\frac{S^{\frac{N}{2}}}{2 N Q_{m}^{\frac{N-2}{2}}}, \frac{S^{\frac{N}{2}}}{N Q_{M}^{\frac{N-2}{2}}}\right) \quad \text { and } \quad \bar{S}_{\infty, h}=\min \left(S_{\infty, h}, \frac{S_{-\mu}^{\frac{N}{2}}}{N Q(0)^{\frac{N-2}{2}}}\right)
$$

Proposition 6.2. (i) Let $0<\mu<\frac{1}{c_{N}}$ and $\lambda \in \mathbb{R}$. Then $I_{-\mu, \lambda}$ satisfies the $(P S)_{c}$ condition for $c<\bar{S}_{\infty, h}$.

(ii) Let $\mu>0$ and $\lambda \in \mathbb{R}$. Then $I_{\mu, \lambda}$ satisfies $(P S)_{c}$ condition for $c<S_{\infty, h}$.

The proof is straightforward application of the concentration-compactness principle and is omitted.

We are now in a position to establish the existence results through a min-max principle based on a topological linking. First we consider problem (24). We assume that

$$
\lambda_{k-1}^{\mu}<\lambda<\lambda_{k}^{\mu} \text { for some } k .
$$

Let $E_{\mu}^{-}=\operatorname{span}\left\{e_{1}^{\mu}, \ldots, e_{l}^{\mu}\right\}$, where $e_{1}^{\mu}, \ldots, e_{l}^{\mu}$ are all eigenfunctions corresponding to eigenvalues $\lambda_{1}^{\mu} \ldots, \lambda_{k-1}^{\mu}$. We have the orthogonal decomposition

$$
H^{1}(\Omega)=E_{\mu}^{-} \oplus E_{\mu}^{+}
$$

Let $w \in E_{\mu}^{+}-\{0\}$ and define a set

$$
M^{\mu}=\left\{u \in H^{1}(\Omega) ; u=v+s w, v \in E_{\mu}^{-}, s \geq 0,\|u\| \leq R\right\} .
$$


Proposition 6.3. There exists $\alpha>0, \rho>0$ and $R>\rho$ ( $R$ depending on $w$ ) such that

$$
I_{\mu, \lambda}(u) \geq \alpha \text { for all } u \in E_{\mu}^{+} \cap \partial B(0, \rho)
$$

and

$$
I_{\mu, \lambda}(u) \leq 0 \text { for } u \in \partial M^{\mu} .
$$

The proof is standard and is omitted.

We now define

$$
Z_{\epsilon}=E_{\mu}^{-} \oplus \mathbb{R} U_{\epsilon, y}=E_{\mu}^{-} \oplus \mathbb{R} U_{\epsilon, y}^{+},
$$

where $U_{\epsilon, y}^{+}$denotes the projection of $U_{\epsilon, y}$ onto $E_{\mu}^{+}$. From now on we use $U_{\epsilon, y}^{+}$in the definition of $M^{\mu}$.

Theorem 6.4. Suppose that $N \geq 5$.

(i) Let $Q_{M} \leq 2^{\frac{2}{N-2}} Q_{m}$. Suppose that (10) and (28) hold. Then problem (24) has a solution.

(ii) Let $Q_{M}>2^{\frac{2}{N-2}} Q_{m}$. Suppose that $Q_{M}=Q(y)$ with $y \neq 0, Q \in C^{2}(B(0, \rho))$ for some ball $B(0, \rho) \subset \Omega$ and $D_{i j} Q(y)=0, i, j=1, \ldots, N$. Then for every $\mu$ there exists an integer $k(\mu) \geq 1$ such that for $\lambda_{j-1}<\lambda<\lambda_{j}$ with $j \geq k(\mu)$, problem (24) has a solution.

Proof. (i) We follow, with some modifications, the argument on pp. 52-53 in [36]. For $u \neq 0$ we have

$$
\max _{t \geq 0} I_{\mu, \lambda}(t u)=\frac{1}{N} \frac{\left(\int_{\Omega}\left(|\nabla u|^{2}+\mu \frac{u^{2}}{|x|^{2}}-\lambda u^{2}\right) d x\right)^{\frac{N}{2}}}{\left(\int_{\Omega} Q(x)|u|^{2^{*}} d x\right)^{\frac{N-2}{2}}}
$$

whenever the integral in the numerator is positive and the maximum is 0 otherwise. In what follows we always denote by $C_{i}$ positive constants independent of $\epsilon$. It is sufficient to show that

$$
m_{\epsilon}^{\mu}=\sup _{u \in Z_{\epsilon},\|u\|_{2^{*}, Q}=1} \int_{\Omega}\left(|\nabla u|^{2}+\mu \frac{u^{2}}{|x|^{2}}-\lambda u^{2}\right) d x<\frac{S}{2^{\frac{2}{N}} Q_{m^{\frac{N-2}{N}}}} .
$$

This obviously implies that

$$
\sup _{u \in M} I_{\mu, \lambda}(u)<\frac{S^{\frac{N}{2}}}{2 N Q_{m^{\frac{N-2}{2}}}} .
$$

If $u \in Z_{\epsilon}$ and $\|u\|_{2^{*}, Q}=1$, then

$$
u=u^{-}+s U_{\epsilon, y}=\left(u^{-}+s U_{\epsilon, y}^{-}\right)+s U_{\epsilon, y}^{+},
$$


where $U_{\epsilon, y}^{-}$and $U_{\epsilon, y}^{+}$denote the projections of $U_{\epsilon, y}$ on $E_{\mu}^{-}$and $E_{\mu}^{+}$, respectively. We now observe that

$$
\int_{\Omega}\left(\left|\nabla U_{\epsilon, y}^{-}\right|^{2}+\frac{\mu}{|x|^{2}}\left(U_{\epsilon, y}^{-}\right)^{2}-\lambda\left(U_{\epsilon, y}^{-}\right)^{2}\right) d x \leq 0
$$

so

$$
\int_{\Omega}\left(\left|\nabla U_{\epsilon, y}^{-}\right|^{2}+\frac{\mu}{|x|^{2}}\left(U_{\epsilon, y}^{-}\right)^{2}\right) d x \leq \int_{\Omega} \lambda\left(U_{\epsilon, y}^{-}\right)^{2} d x \leq \lambda \int_{\Omega} U_{\epsilon, y}^{2} d x=O\left(\epsilon^{2}\right) .
$$

By the Sobolev inequality, we deduce

$$
\left\|U_{\epsilon, y}^{-}\right\|_{2^{*}}^{2} \leq C_{1} \int_{\Omega}\left(\left|\nabla U_{\epsilon, y}^{-}\right|^{2}+\mu r\left(U_{\epsilon, y}^{-}\right)^{2}\right) d x \leq C_{1} \int_{\Omega}\left(\left|\nabla U_{\epsilon, y}^{-}\right|^{2}+\frac{\mu}{|x|^{2}}\left(U_{\epsilon, y}^{-}\right)^{2}\right) d x \rightarrow 0
$$

as $\epsilon \rightarrow 0$. Therefore there exists a constant $C_{2}>0$ such that $0<s \leq C_{2}$ and $\left\|u^{-}\right\|_{2^{*}} \leq C_{2}$. It follows from the convexity of $\|\cdot\|_{2^{*}, Q}^{2^{*}}$ that

$$
\begin{aligned}
1=\|u\|_{2^{*}, Q}^{2^{*}} & \geq\left\|s U_{\epsilon, y}\right\|_{2^{*}, Q}^{2^{*}}+2^{*} \int_{\Omega} Q u^{-}\left(s U_{\epsilon, y}\right)^{2^{*}-1} d x \\
& \geq\left\|s U_{\epsilon, y}\right\|_{2^{*}, Q}^{2^{*}}-C_{3}\left\|U_{\epsilon, y}\right\|_{2^{*}-1}^{2^{*}-1}\left\|u^{-}\right\|_{2} .
\end{aligned}
$$

Hence

$$
\left\|s U_{\epsilon, y}\right\|_{2^{*}, Q}^{2^{*}} \leq C_{4} \epsilon^{\frac{N-2}{2}}+1
$$

Since all norms in $E_{\mu}^{-}$are equivalent, we see that

$$
\begin{aligned}
& \int_{\Omega}\left(\nabla u^{-} \nabla U_{\epsilon, y}+\frac{\mu}{|x|^{2}} u^{-} U_{\epsilon, y}\right) d x \leq \\
& \quad \leq C_{5}\left(\left\|\nabla U_{\epsilon, y}\right\|_{1}+\left\|\frac{1}{|\cdot|^{2}} U_{\epsilon, y}\right\|_{1}\right)\left\|u^{-}\right\|_{2}=O\left(\epsilon^{\frac{N-2}{2}}\right)\left\|u^{-}\right\|_{2} .
\end{aligned}
$$

It follows from (10) that

$$
\left\|U_{\epsilon, y}\right\|_{2^{*} Q}^{2^{*}}=Q_{m} \int_{\Omega} U_{\epsilon, y}^{2^{*}} d x+o(\epsilon)
$$


With the aid of (29) we obtain

$$
\begin{aligned}
\int_{\Omega}\left(|\nabla u|^{2}\right. & \left.+\frac{\mu}{|x|^{2}} u^{2}-\lambda u^{2}\right) d x \\
& \leq\left(\lambda_{k-1}^{\mu}-\lambda\right) \int_{\Omega}\left|u^{-}\right|^{2} d x+O\left(\epsilon^{\frac{N-2}{2}}\right)\left\|u^{-}\right\|_{2} \\
& +s^{2} \int_{\Omega}\left(\left|\nabla U_{\epsilon, y}\right|^{2}+\mu \frac{U_{\epsilon, y}^{2}}{|x|^{2}}-\lambda U_{\epsilon, y}^{2}\right) d x \\
& =-\left(\lambda-\lambda_{k-1}^{\mu}\right)\left\|u^{-}\right\|_{2}^{2}+O\left(\epsilon^{\frac{N-2}{2}}\right)\left\|u^{-}\right\|_{2} \\
& +s^{2} \int_{\Omega}\left(\left|\nabla U_{\epsilon, y}\right|^{2}+\mu \frac{U_{\epsilon, y}^{2}}{|x|^{2}}-\lambda U_{\epsilon, y}^{2}\right) d x \\
& =-\left(\lambda-\lambda_{k-1}^{\mu}\right)\left\|u^{-}\right\|_{2}^{2}+O\left(\epsilon^{\frac{N-2}{2}}\right)\left\|u^{-}\right\|_{2} \\
& +\frac{\int_{\Omega}\left(\left|\nabla U_{\epsilon, y}\right|^{2}+\mu \frac{U_{\epsilon, y}^{2}}{|x|^{2}}-\lambda U_{\epsilon, y}^{2}\right) d x}{\left(\int_{\Omega} Q(x) U_{\epsilon, y}^{2^{*}} d x\right)^{\frac{2}{2^{*}}}}\left(s^{2^{*}} \int_{\Omega} Q(x) U_{\epsilon, y}^{2^{*}} d x\right)^{\frac{2}{2^{*}}}
\end{aligned}
$$

We now take into account the estimate (9), in order to estimate the ratio term on the right hand side of (30). We then have

$$
\begin{aligned}
m_{\epsilon}^{\mu} & \leq-\left(\lambda-\lambda_{k-1}^{\mu}\right)\left\|u^{-}\right\|_{2}^{2}+O\left(\epsilon^{\frac{N-2}{2}}\left\|u_{-}\right\|_{2}\right) \\
& +\left(\frac{S}{2 \frac{2}{N} Q_{m}^{\frac{N-2}{N}}}-\epsilon A_{N} Q_{m^{\frac{N-2}{N}}} H(y)+o(\epsilon)\right)\left(1+C_{4} \epsilon^{\frac{N-2}{2}}\right)
\end{aligned}
$$

for some constant $A_{N}>0$ and the result follows.

(ii) The only change is in the estimating the ratio term on the right-hand side of (30). First we observe that

$$
\int_{\Omega} \frac{1}{|x|^{2}} U_{\epsilon, y}^{2} d x=O\left(\epsilon^{2}\right) \quad \text { and } \quad \int_{\Omega} U_{\epsilon, y}^{2} d x \geq c_{1} \epsilon^{2}
$$

for some $c_{1}>0$ independent of $\epsilon>0$. Moreover, we have

$$
\int_{\Omega} Q(x) U_{\epsilon, y}^{2^{*}} d x=Q_{M} \int_{\Omega} U_{\epsilon, y}^{2^{*}} d x+o\left(\epsilon^{2}\right) .
$$

Since $\lim _{j \rightarrow \infty} \lambda_{j}^{\mu}=\infty$, we can find an integer $k(\mu)$ such for $\lambda_{j-1}^{\mu}<\lambda<\lambda_{j}$ and $j \geq k(\mu)$ the term $\lambda \int_{\Omega} U_{\epsilon, y}^{2} d x$ dominates $\mu \int_{\Omega} \frac{1}{|x|^{2}} U_{\epsilon, y}^{2} d x$ and the result follows.

We now consider problem (25). We use similar notations as in the case (24). By $\left\{\left(e_{j}^{-\mu}\right\}, j=1,2, \ldots\right.$, we denote the sequence of eigenfunctions corresponding to eigenvalues $\left\{\lambda_{j}^{-\mu}\right\}, j=1,2, \ldots$. We assume that a parameter $\lambda$ satisfies

$$
\lambda_{k-1}^{-\mu}<\lambda<\lambda_{k}^{-\mu} \text { for some } k .
$$


We set $E_{-\mu}^{-}=\operatorname{span}\left\{e_{1}^{-\mu}, \ldots, e_{l}^{-\mu}\right\}$, where $e_{1}^{-\mu}, \ldots, e_{l}^{-\mu}$ are eigenfunctions corresponding the eigenvalues $\lambda_{1}^{-\mu}, \ldots, \lambda_{k-1}^{-\mu}$. We have the orthogonal decomposition $H^{1}(\Omega)=E_{-\mu}^{-} \oplus E_{-\mu}^{+}$. Let $w \in E_{-\mu}^{-}-\{0\}$ and define a set

$$
M^{-\mu}=\left\{u \in H^{1}(\Omega) ; u=v+s w, v \in E_{-\mu}^{-}, s \geq 0, \text { and }\|u\| \leq R\right\} .
$$

Proposition 6.5. Suppose that (31) holds. Then there exists $\alpha>0, \rho>0$ and $R>\rho$ (depending on $w$ ) such that

$$
I_{-\mu, \lambda}(u) \geq \alpha \text { for every } u \in E_{-\mu}^{+} \cap \partial B(0, \rho)
$$

and

$$
I_{-\mu, \lambda}(u) \leq 0 \text { for every } u \in \partial M^{-\mu} .
$$

Theorem 6.6. Suppose that $N \geq 5$.

(i) Let $\bar{S}_{\infty, h}=\frac{S^{\frac{N}{2}}}{2 N Q_{m^{2}}^{\frac{N-2}{2}}}$. Suppose that for some $y \in \partial \Omega$ with $H(y)>0$ and $Q_{m}=Q(y)$ we have

$$
|Q(y)-Q(x)|=o(|x-y|) \text { for } x \text { close to } y .
$$

If $\lambda$ satisfies (31), then problem (25) has a solution.

(ii) Let $\bar{S}_{\infty, h}=\frac{S^{\frac{N}{2}}}{N Q_{M}^{\frac{N-2}{2}}}$. Suppose that $Q(y)=Q_{M}$ with $y \neq 0, Q \in C^{2}(B(0, \rho))$ for some ball $B(0, \rho) \subset \Omega$ and $D_{i j} Q(0)=0, i, j=1, \ldots, N$. If $\lambda>0$ and satisfies (31), then problem (25) has a solution.

(iii) Let $\bar{S}_{\infty, h}=\frac{S_{-\mu}^{\frac{N}{2}}}{N Q(0)^{\frac{N-2}{2}}}$ and $Q \in C^{2}(B(0, \rho))$. Suppose that (31) holds and $\mu<\bar{\mu}-1$ and $\lambda>0$ and moreover $D_{i} Q(0)=0$ and $D_{i j} Q(0)=0, i, j=1, \ldots, N$. Then problem (25) has a solution.

Proof. The proof of (i) and (ii) is similar to the proof Theorem 24. In both cases we

use $w=U_{\epsilon, y}^{+}$in the definition of $M^{-\mu}$.
$\quad$ To show that $\sup _{u \in M^{-\mu}} I_{-\mu, \lambda}(u)<\frac{S_{-\mu}^{\frac{N}{2}}}{N Q(0)^{\frac{N-2}{2}}}$ we take $w=U_{\epsilon}^{\mu}$ in the definition of $M^{-\mu}$. By straightforward calculations we verify that

$$
a \epsilon^{2} \leq \int_{\Omega}\left(U_{\epsilon}^{\mu}\right)^{2} d x \leq b \epsilon^{2}
$$

for some constants $a>0$ and $b>0$ independent of $\epsilon$. Moreover, if $\mu<\bar{\mu}-1$, then

$$
\int_{\Omega}\left(\left|\nabla U_{\epsilon}^{\mu}\right|^{2}-\frac{\mu}{|x|^{2}}\left(U_{\epsilon}^{\mu}\right)\right) d x=\int_{\mathbb{R}^{N}}\left(\left|\nabla U_{\epsilon}^{\mu}\right|^{2}-\frac{\mu}{|x|^{2}}\left(U_{\epsilon}^{\mu}\right)\right) d x+o\left(\epsilon^{2}\right)
$$


and

$$
\int_{\Omega}\left(U_{\epsilon}^{\mu}\right)^{2^{*}} d x=\int_{\mathbb{R}^{N}}\left(U_{\epsilon}^{\mu}\right)^{2^{*}} d x+o\left(\epsilon^{2}\right) .
$$

These estimates allow to derive the following inequality

$$
\frac{\int_{\Omega}\left(\left|\nabla U_{\epsilon}^{\mu}\right|^{2}-\frac{\mu}{|x|^{2}}\left(U_{\epsilon}^{\mu}\right)^{2}-\lambda\left(U_{\epsilon}^{\mu}\right)^{2}\right) d x}{\left(\int_{\Omega} Q(x)\left(U_{\epsilon}^{\mu}\right)^{2^{*}} d x\right)^{\frac{2}{2^{*}}}}<\frac{S_{-\mu}^{\frac{N}{2}}}{Q(0)^{\frac{N-2}{N}}}-\lambda \bar{a} \epsilon^{2}
$$

for some constant $\bar{a}>0$. This obviously implies the desired estimate form above of $I_{-\mu, \lambda}$ on the set $Z^{-\mu}$.

\section{Critical Hardy-Sobolev nonlinearity}

In this section we are concerned with the existence of solutions of the following problem

$$
\begin{cases}-\Delta u-\frac{\mu}{|x|^{\alpha}}|u|^{2_{\alpha}^{*}-2} u+\lambda u & =Q(x)|u|^{q-2} u \text { in } \Omega, \\ \frac{\partial}{\partial \nu} u(x) & =0 \text { on } \partial \Omega .\end{cases}
$$

We assume that $\lambda>0$ and $\mu>0$. For $0 \leq \alpha<2,2_{\alpha}^{*}=2 \frac{N-\alpha}{N-2}$ is the limiting exponent for the Hardy-Sobolev embedding $H_{\circ}^{1}(\Omega) \rightarrow L^{2_{\alpha}^{*}}\left(\Omega,|x|^{-\alpha}\right)$ and $2<q \leq 2^{*}$. It is known that that $H_{\circ}^{1}(\Omega)$ is continuously embedded into $L^{2_{\alpha}^{*}}\left(\Omega,|x|^{-\alpha}\right)$. If $2 \leq p<2_{\alpha}^{*}$, then $H_{\circ}^{1}(\Omega)$ is compactly embedded into $L^{p}\left(\Omega,|x|^{-\alpha}\right)$. Let

$$
S_{\alpha}=\inf _{u \in H_{\circ}^{1}(\Omega)-\{0\}} \frac{\int_{\Omega}|\nabla u|^{2} d x}{\left(\int_{\Omega} \frac{|u|^{2 *}}{|x|^{\alpha}} d x\right)^{\frac{2}{2_{\alpha}^{*}}}} .
$$

The constant $S_{\alpha}$ is independent of $\Omega$ and is not achieved if $\Omega \neq \mathbb{R}^{N}$. If $\alpha=0$, then $S_{\circ}=S$. For every $\epsilon>0$, the family of functions

$$
u_{\epsilon}(x)=\frac{\epsilon^{\frac{N-2}{2}}((N-2)(N-\alpha))^{\frac{N-2}{2(2-\alpha)}}}{\left(\epsilon^{2-\alpha}+|x|^{2-\alpha}\right)^{\frac{N-2}{2-\alpha}}}
$$

satisfies the equation

$$
-\Delta u_{\epsilon}=|x|^{-\alpha} u_{\epsilon}^{2_{\alpha}^{*}} \text { in } \mathbb{R}^{N}
$$

and is a minimizer for

$$
S_{\alpha}=\inf _{u \in H^{1}\left(\mathbb{R}^{N}\right)} \frac{\int_{\mathbb{R}^{N}}|\nabla u|^{2} d x}{\left(\int_{\mathbb{R}^{N}}|u|^{2_{\alpha}^{*}}|x|^{-\alpha} d x\right)^{\frac{2}{2 *}}} .
$$

We also have

$$
\int_{\mathbb{R}^{N}}\left|\nabla u_{\epsilon}(x)\right|^{2} d x=\int_{\mathbb{R}^{N}} u_{\epsilon}(x)^{2_{\alpha}^{*}}|x|^{-\alpha} d x=S_{\alpha}^{\frac{N-\alpha}{N-2}}
$$


It follows from (33) that

$$
S_{\alpha}\left(\int_{\Omega} \frac{|u|^{2_{\alpha}^{*}}}{|x|^{\alpha}}\right)^{\frac{2}{2_{\alpha}^{*}}} d x \leq \int_{\Omega}|\nabla u|^{2} d x
$$

for every $u \in H_{\circ}^{1}(\Omega)$. It is clear that this inequality is no longer true in $H^{1}(\Omega)$. As in Section 2 we formulate the following modification of this inequality in $H^{1}(\Omega)$.

Lemma 7.1. For every $\delta>0$ there exists a constant $C(\delta)>0$ such that

$$
\left(\int_{\Omega} \frac{|u|^{2_{\alpha}^{*}}}{|x|^{\alpha}} d x\right)^{\frac{2}{2_{\alpha}^{*}}} \leq\left(S_{\alpha}^{-1}+\delta\right) \int_{\Omega}|\nabla u|^{2} d x+C(\delta)\left[\int_{\Omega} u^{2} d x+\left(\int_{\Omega}|u|^{2_{\alpha}^{*}} d x\right)^{\frac{2}{2_{\alpha}^{*}}}\right]
$$

Proof. Let $\phi$ be a function defined in the proof of Lemma 2.1. Then

$$
\begin{gathered}
\left(\int_{\Omega} \frac{|u|^{2_{\alpha}^{*}}}{|x|^{\alpha}} d x\right)^{\frac{2}{2_{\alpha}^{*}}} \leq\left(\int_{\Omega} \frac{|u \phi|^{2_{\alpha}^{*}}}{|x|^{\alpha}} d x\right)^{\frac{2}{2_{\alpha}^{*}}}+\left(\int_{\Omega} \frac{|u|^{2_{\alpha}^{*}}\left(1-\phi^{2_{\alpha}^{*}}\right)}{|x|^{\alpha}} d x\right)^{\frac{2}{2_{\alpha}^{*}}} \\
\leq S_{\alpha}^{-1} \int_{\Omega}\left(|\nabla u|^{2} \phi^{2}+2 u \nabla u \phi \nabla \phi+u^{2}|\nabla \phi|^{2}\right) d x+C_{1}\left(\int_{\Omega}|u|^{2_{\alpha}^{*}} d x\right)^{\frac{2}{2_{\alpha}^{*}}} .
\end{gathered}
$$

for some constant $C_{1}>0$. An application of the Young inequality completes the proof.

Solutions of (32) will be sought as critical points of the functional

$$
J_{\alpha,-\mu}(u)=\frac{1}{2} \int_{\Omega}\left(|\nabla u|^{2}+\lambda u^{2}\right) d x-\frac{\mu}{2_{\alpha}^{*}} \int_{\Omega} \frac{|u|^{2_{\alpha}^{*}}}{|x|^{\alpha}} d x-\frac{1}{q} \int_{\Omega} Q(x)|u|^{q} d x .
$$

It follows from Lemma 7.1 that the functional $J_{\alpha,-\mu}(u)$ is well defined for $u \in H^{1}(\Omega)$. It is easy to verify that $J_{\alpha,-\mu}$ is $C^{1}$ and

$$
\left\langle J_{\alpha,-\mu}^{\prime}(u), \phi\right\rangle=\int_{\Omega}(\nabla u \nabla \phi+\lambda u \phi) d x-\mu \int_{\Omega} \frac{|u|^{2_{\alpha}^{*}-2} u}{|x|^{\alpha}} \phi d x-\int_{\Omega} Q(x)|u|^{q-2} u \phi d x
$$

for every $\phi \in H^{1}(\Omega)$.

We set

$$
S_{\infty, \alpha,-\mu}=\min \left(\frac{S^{\frac{N}{2}}}{N Q_{M}^{\frac{N-2}{2}}}, \frac{S^{\frac{N}{2}}}{2 N Q^{\frac{N-2}{2}}}, \frac{S^{\frac{N}{2}}}{N(Q(0)+\mu)^{\frac{N-2}{2}}}, \frac{(2-\alpha) \mu S_{\alpha}^{\frac{N-\alpha}{2-\alpha}}}{2(N-\alpha)(Q(0)+\mu)^{\frac{N-2}{2-\alpha}}}\right)
$$

Proposition 7.2. (i) If $q=2^{*}$, then the functional $J_{\alpha,-\mu}$ satisfies the $(P S)_{c}$ condition for $c<S_{\infty, \alpha,-\mu}$. 
(ii) If $2<q<2^{*}$, then the functional $J_{\alpha,-\mu}$ satisfies the $(P S)_{c}$ condition for

$$
c<\frac{(2-\alpha)}{2(N-\alpha)} \frac{S^{\frac{N}{2}}}{(Q(0)+\mu)^{\frac{N-2}{2-\alpha}}} .
$$

Proof. (i) Let $\left\{u_{m}\right\}$ be a $(P S)_{c}$ sequence. Then

$$
\begin{aligned}
J_{\alpha,-\mu}\left(u_{m}\right) & -\frac{1}{2}\left\langle J_{\alpha,-\mu}^{\prime}\left(u_{m}\right), u_{m}\right\rangle \\
& =\frac{(2-\alpha) \mu}{2(N-\alpha)} \int_{\Omega} \frac{\left|u_{m}\right|^{2_{\alpha}^{*}}}{|x|^{\alpha}} d x+\frac{1}{N} \int_{\Omega} Q(x)\left|u_{m}\right|^{2^{*}} d x \leq c+1+o\left(\left\|u_{m}\right\|\right)
\end{aligned}
$$

for $m \geq m_{\circ}$. This combined with the fact that $J_{\alpha,-\mu}\left(u_{m}\right) \rightarrow c$ implies that the sequence $\left\{u_{m}\right\}$ is bounded in $H^{1}(\Omega)$. Since $\left\{u_{m}\right\}$ is bounded in $H^{1}(\Omega)$ we may assume that $u_{m} \rightarrow u$ in $H^{1}(\Omega), L^{2^{*}}(\Omega), L_{\alpha}^{2_{\alpha}^{*}}\left(\Omega,|x|^{-\alpha}\right)$ and $u_{m} \rightarrow u$ in $L^{p}(\Omega)$ for $2 \leq p<\infty$. On the other hand by the concentration - compactness principle we have

$$
\left|u_{n}\right|^{2^{*}} \stackrel{*}{\rightarrow}|u|^{2^{*}}+\sum_{j \in J} \nu_{j} \delta_{x_{j}}+\nu_{\circ} \delta_{\circ}
$$

and

$$
\left|\nabla u_{m}\right|^{2} \stackrel{*}{\rightarrow}|\nabla u|^{2}+\sum_{j \in J} \mu_{j} \delta_{x_{j}}+\mu_{\circ} \delta_{\circ} .
$$

The sequence $\left\{\frac{\left|u_{m}\right|_{\alpha}^{*}}{|x|^{\alpha}}\right\}$ can only concentrate at 0 , so we have

$$
\frac{\left|u_{m}\right|^{2_{\alpha}^{*}}}{|x|^{\alpha}} \stackrel{*}{\rightarrow} \frac{|u|^{2_{\alpha}^{*}}}{|x|^{\alpha}}+\bar{\nu}_{\circ} .
$$

Using a family of test functions concentrating at $x_{j}$ (or at 0 ), we derive the following relations

$$
\mu_{j}=Q\left(x_{j}\right) \nu_{j} \text { for } x_{j} \neq 0
$$

and

$$
\mu_{\circ}=Q(0) \nu_{\circ}+\mu \bar{\nu}_{\circ}
$$

We now show that all coefficients $\nu_{j}$ and $\bar{\nu}_{\circ}$ vanish. If $\nu_{j}>0$ for some $x_{j} \in \Omega$, then by (35) and the fact that $S \nu_{j}^{\frac{2}{2 *}} \leq \mu_{j}$ we get that

$$
\nu_{j} \geq \frac{S^{\frac{N}{2}}}{Q\left(x_{j}\right)^{\frac{N}{2}}} .
$$

If $x_{j} \in \partial \Omega$, then $\frac{S}{2^{\frac{2}{N}}} \nu_{j}^{\frac{2}{2^{*}}} \leq \mu_{j}$ and

$$
\nu_{j} \geq \frac{S^{\frac{N}{2}}}{2 Q\left(x_{j}\right)^{\frac{N}{2}}} .
$$


Assuming that $\nu_{j}>0$ for $x_{j} \in \Omega$, then

$$
c=\lim _{m \rightarrow \infty}\left[J_{\alpha,-\mu}\left(u_{m}\right)-\frac{1}{2}\left\langle J_{\alpha,-\mu}\left(u_{m}\right), u_{m}\right\rangle\right] \geq \frac{1}{N} Q\left(x_{j}\right) \nu_{j} \geq \frac{S^{\frac{N}{2}}}{N Q_{m^{\frac{N-2}{2}}}}
$$

and we get a contradiction. In a similar manner we show that $\nu_{j}=0$ if $x_{j} \in \partial \Omega$. We now distinguish two cases: (a) $\nu_{\circ}<\bar{\nu}_{\circ}$ and (b) $\bar{\nu}_{\circ} \leq \nu_{\circ}$. If (a) occurs, then $\mu_{\circ} \leq(Q(0)+\mu) \bar{\nu}_{\circ}$ and by (33) we get

$$
\bar{\nu}_{\circ} \geq \frac{S_{\alpha}^{\frac{N-2}{2-\alpha}}}{(Q(0)+\mu)^{\frac{N-\alpha}{2-\alpha}}} .
$$

Since

$$
\begin{aligned}
& c>\lim _{m \rightarrow \infty}\left[J_{\alpha,-\mu}\left(u_{m}\right)-\frac{1}{2}\left\langle J_{\alpha,-\mu}\left(u_{m}\right), u_{m}\right\rangle\right] \\
& \geq \frac{(2-\alpha) \mu S_{\alpha}^{\frac{N-\alpha}{2-\alpha}}}{2(N-\alpha)(Q(0)+\mu)^{\frac{N-\alpha}{2-\alpha}}}
\end{aligned}
$$

we have arrived at a contradiction. If (b) prevails, then $\mu_{\circ} \leq(Q(0)+\mu) \nu_{\circ}$ and consequently

$$
\nu_{\circ}>\frac{S^{\frac{N}{2}}}{(Q(0)+\mu)^{\frac{N-2}{2}}} .
$$

Again, as in the previous case, we get a contradiction.

To obtain critical points of $J_{\alpha,-\mu}$ we apply the mountain-pass principle. First we check that the functional $J_{\alpha,-\mu}$ has a mountain-pass geometry. It follows from Lemma 7.1 and Sobolev inequalities that

$$
\begin{aligned}
J_{\alpha,-\mu}(u) & \geq \frac{\min (1, \lambda)}{2} \int_{\Omega}\left(|\nabla u|^{2}+u^{2}\right) d x \\
& -\frac{\mu}{2_{\alpha}^{*}}\left[\left(S_{\alpha}^{-1}+\delta\right) \int_{\Omega}\left(|\nabla u|^{2}+\lambda u^{2}\right) d x+\tilde{C}(\delta) \int_{\Omega}\left(|\nabla u|^{2}+u^{2}\right) d x\right]^{\frac{2_{\alpha}^{*}}{2}} \\
& -C_{1}\left(\int_{\Omega}\left(|\nabla u|^{2}+u^{2}\right) d x\right)^{\frac{q}{2}}
\end{aligned}
$$

for some constants $\tilde{C}(\delta)>0$ and $C_{1}>0$. Since $2_{\alpha}^{*}>2$, we can choose constants $\rho>0$ and $\alpha>0$ so that

$$
J_{\alpha,-\mu}(u) \geq \alpha \text { for }\|u\|=\rho .
$$


For every $v \neq 0$ in $H^{1}(\Omega)$ we have $J_{\alpha,-\mu}(t v)<0$ and $\|t v\|>\rho$ for sufficiently large $t>0$. We now define the mountain-pass level

$$
d_{\alpha,-\mu}=\inf _{\gamma \in \Gamma} \max _{0 \leq t \leq 1} J_{\alpha,-\mu}(\gamma(t))
$$

where

$$
\Gamma=\left\{\gamma \in C\left([0,1], H^{1}(\Omega)\right) ; \gamma(0)=(0), \gamma(1)=v\right\}
$$

with $\|v\|>\rho$ and $J_{\alpha,-\mu}(v)<0$.

First we establish an existence result in the subcritical case.

Theorem 7.3. Suppose that $2<q<2^{*}$ if $N \geq 4$ and $4<q<6$ if $N=3$. Then for every $\lambda>$ and $\mu>0$ problem (32) admits a solution.

Proof. According to Proposition 7.2 we must show that

$$
d_{\alpha,-\mu}<\frac{(2-\alpha) S^{\frac{N-\alpha}{2-\alpha}}}{2(N-\alpha) \mu^{\frac{N-\alpha}{2-\alpha}}}
$$

We take $v=u_{\epsilon}$ in the definition of the mountain-pass level. Since $\lim _{t \rightarrow \infty} J_{\alpha,-\mu}\left(t u_{\epsilon}\right)=$ $-\infty$, there exists $t_{\epsilon}>0$ such that

$$
J_{\alpha,-\mu}\left(t_{\epsilon}, u_{\epsilon}\right)=\max _{t \geq 0} J_{\alpha,-\mu}\left(t u_{\epsilon}\right)
$$

and

$$
t_{\epsilon}\left\|u_{\epsilon}\right\|^{2}-\mu t_{\epsilon}^{2_{\alpha}^{*}-1} \int_{\Omega} \frac{u_{\epsilon}^{2_{\alpha}^{*}}}{|x|^{\alpha}} d x=t_{\epsilon}^{2_{\alpha}^{*}-1} \int_{\Omega} Q(x) u_{\epsilon}^{2^{*}-1} d x
$$

Hence

$$
t_{\epsilon} \leq\left(\frac{\left\|u_{\epsilon}\right\|^{2}}{\mu \int_{\Omega} \frac{u_{\epsilon}^{2 *}}{|x|^{\alpha}} d x}\right)^{\frac{1}{2 \alpha_{\alpha}^{*}-2}}
$$

and

$$
J_{\alpha,-\mu}\left(t_{\epsilon} u_{\epsilon}\right) \leq \frac{(2-\alpha)}{2(N-\alpha)} \frac{\left(\left\|u_{\epsilon}\right\|^{2}\right)^{\frac{2_{\alpha}^{*}}{2 \alpha}-2}}{\left(\mu \int_{\Omega} \frac{u_{\epsilon}^{2 *}}{|x|^{\alpha}} d x\right)^{\frac{2}{2 *}}}-\frac{t_{\epsilon}^{q}}{q} \int_{\Omega} Q(x) u_{\epsilon}^{q} d x
$$

Since

$$
\int_{\Omega}\left(\left|\nabla u_{\epsilon}\right|^{2}+\lambda u_{\epsilon}^{2}\right) d x=S_{\alpha}^{\frac{N-\alpha}{2-\alpha}}+O\left(\epsilon^{N-\alpha}\right)+\lambda O\left(\epsilon^{2}\right)
$$

and

$$
\int_{\Omega} \frac{u_{\epsilon}^{2_{\alpha}^{*}}}{|x|^{\alpha}} d x=S_{\alpha}^{\frac{N-\alpha}{2-\alpha}}+O\left(\epsilon^{N-\alpha}\right)
$$


we deduce from (40) that

$$
J_{\alpha,-\mu}\left(t_{\epsilon} u_{\epsilon}\right) \leq \frac{2-\alpha}{2(N-\alpha)} \frac{S_{\alpha}^{\frac{N-\alpha}{2-\alpha}}}{\mu^{\frac{N-2}{2-\alpha}}}+\lambda O\left(\epsilon^{2}\right)-t_{\epsilon}^{q} \int_{\Omega} Q(x) u_{\epsilon}^{q} d x .
$$

We now verify that

$$
\int_{\Omega} Q(x) u_{\epsilon}^{q} d x \geq b \epsilon^{N-q \frac{N-2}{2}}
$$

provided $\frac{N}{N-2}<q$. This condition is satisfied if $2<q$ for $N \geq 4$ and $3<q$ for $N=3$. In both cases we have $N-q \frac{N-2}{2}<2$. Hence

$$
\max _{t \geq 0} J_{\alpha,-\mu}\left(t u_{\epsilon}\right)<\frac{(2-\alpha)}{2(N-\alpha)} \frac{S_{\alpha}^{\frac{N-\alpha}{2-\alpha}}}{\mu^{\frac{N-2}{2-\alpha}}}
$$

for $\epsilon>0$ sufficiently small. This completes the proof of (39) and the result follows from the mountain-pass theorem.

The assertion of Proposition 7.2 becomes more transparent if $Q(0)=0$ (we assume that $Q(x)>0$ for $x \neq 0)$.

Proposition 7.4. Let $q=2^{*}$ and $Q(0)=0$. Then $J_{\alpha,-\mu}$ satisfies the $(P S)_{c}$ condition for

$$
c<\min \left(\frac{S^{\frac{N}{2}}}{N Q_{M}^{\frac{N-2}{2}}}, \frac{S^{\frac{N}{2}}}{2 N Q_{m^{\frac{N-2}{2}}}}, \frac{(2-\alpha) S_{\alpha}^{\frac{N-\alpha}{2-\alpha}}}{2(N-\alpha) \mu^{\frac{N-2}{2-\alpha}}}\right) .
$$

We set

$$
S_{\infty, \alpha,-\mu}^{\circ}=\min \left(\frac{S^{\frac{N}{2}}}{N Q_{M}^{\frac{N-2}{2}}}, \frac{S^{\frac{N}{2}}}{2 N Q_{m^{2}}^{\frac{N-2}{2}}}, \frac{(2-\alpha) S_{\alpha}^{\frac{N-\alpha}{2-\alpha}}}{2(N-\alpha) \mu^{\frac{N-2}{2-\alpha}}}\right) .
$$

We now consider two cases:

(i) there exists a constant $\mu_{\circ}=\mu_{\circ}\left(N, Q_{m}, Q_{M}\right)>0$ such that

$$
S_{\infty, \alpha,-\mu}^{\circ}=\frac{(2-\alpha) S_{\alpha}^{\frac{N-\alpha}{2-\alpha}}}{2(N-\alpha) \mu^{\frac{N-2}{2-\alpha}}}
$$

for $\mu \geq \mu_{\circ}$.

(ii) there exists a constant $\mu_{1}=\mu_{1}\left(N, Q_{M}, Q_{m}\right)$ such that

$$
\min \left(\frac{S^{\frac{N}{2}}}{N Q_{M}^{\frac{N-2}{2}}}, \frac{S^{\frac{N}{2}}}{2 N Q_{m^{\frac{N-2}{2}}}^{2}}\right)=S_{\infty, \alpha,-\mu}^{\circ}
$$

for $0<\mu \leq \mu_{1}$. 
Theorem 7.5. Let $q=2^{*}$ and $Q(0)=0$.

(i) Suppose that (41) holds for $\mu \geq \mu_{\circ}$. Moreover we assume that $Q$ is $C^{2}$ in small ball around 0 and that the Hessian $\left\{D_{i j} Q(0)\right\}$ is positive definite. Then there exists $\bar{\lambda}=\bar{\lambda}(\mu)>0$ such that problem (32) has a solution for $\mu \geq \mu_{\circ}$ and $0<\lambda \leq \bar{\lambda}$.

(ii) Suppose that (42) holds for $0<\mu \leq \mu_{1}$ and let $\alpha<1$. If $Q_{M}>2^{\frac{2}{N-2}} Q_{m}$, $|Q(x)-Q(y)|=o(|x-y|)$ for $x$ near $y$ with $Q(y)=Q_{M}$, then problem (32) has a solution for $0<\mu \leq \mu_{1}$ and $\lambda>0$.

(iii) Suppose that (42) holds for $0<\mu \leq \mu_{1}$. If $Q_{M} \leq 2^{\frac{2}{N-2}},|Q(x)-Q(y)|=$ $o(|x-y|)$ for $x$ near $y$ with $Q(y)=Q_{m}$ and $H(y)>0$, then problem (32) has a solution for $\lambda>0$ and $0<\mu \leq \mu_{1}$.

Proof. (i) We proceed as in the proof of Theorem 7.3. There exists $t_{\epsilon}>0$ such that

$$
J_{\alpha,-\mu}\left(t_{\epsilon} u\right)=\max _{0 \leq t} J_{\alpha,-\mu}\left(t u_{\epsilon}\right) \leq \frac{(2-\alpha)}{2(N-\alpha)} \frac{\left(\left\|u_{\epsilon}\right\|\right)^{\frac{2_{\alpha}^{*}}{2_{\alpha}^{*}-2}}}{\left(\mu \int_{\Omega} \frac{u_{\epsilon}^{\alpha^{*}}}{|x|^{\alpha}} d x\right)^{\frac{2_{\alpha}^{2}}{2^{*}-2}}}-t_{\epsilon}^{2^{*}} \int_{\Omega} Q(x) u_{\epsilon}^{2^{*}} d x
$$

Since $\left\{D_{i j} Q(0)\right\}$ is positive definite, we see that

$$
J_{\alpha,-\mu}\left(t_{\epsilon}, u_{\epsilon}\right) \leq \frac{(2-\alpha) S_{\alpha}^{\frac{N-\alpha}{2-\alpha}}}{2(N-\alpha) \mu^{\frac{N-2}{2-\alpha}}}+\lambda O\left(\epsilon^{2}\right)-c \epsilon^{2}
$$

for some constant $c>0$. We can now find a constant $\bar{\lambda}=\bar{\lambda}(\mu)$ such that for $0<\lambda \leq \bar{\lambda}$ and $\mu>\mu_{\circ}$

$$
J_{\alpha,-\mu}\left(t_{\epsilon}, u_{\epsilon}\right)<\frac{(2-\alpha) S_{\alpha}^{\frac{N-\alpha}{2-\alpha}}}{2(N-\alpha) \mu^{\frac{N-2}{2-\alpha}}} .
$$

(ii) We take $v=U_{\epsilon, y}$ with $Q(y)=Q_{M}$ in the definition of the mountain-pass level. First we observe that

$$
\int_{\Omega} \frac{U_{\epsilon, y}^{2_{\alpha}^{*}}}{|x|^{\alpha}} d x \geq b \epsilon^{\alpha}
$$

for some constant $b>0$. We then have

$$
\begin{aligned}
J_{\alpha,-\mu}\left(\bar{t}_{\epsilon} U_{\epsilon, y}\right) & \leq \frac{1}{N} \frac{\left(\int_{\Omega}\left(\left|\nabla U_{\epsilon, y}\right|^{2}+\lambda U_{\epsilon, y}^{2}\right) d x\right)^{\frac{2^{*}}{2^{*}-2}}}{\left(\int_{\Omega} Q(x) U_{\epsilon, y}^{2^{*}} d x\right)^{\frac{2}{2^{*}-2}}}-\frac{\mu}{2_{\alpha}^{*}} \int_{\Omega} \frac{U_{\epsilon, y}^{2_{\alpha}^{*}}}{|x|^{\alpha}} d x \\
& =\frac{\left(S^{\frac{N}{2}}+\lambda O\left(\epsilon^{2}\right)\right)^{\frac{2}{2^{*}-2}}}{\left(Q_{M} S^{\frac{N}{2}}+o(\epsilon)\right)^{\frac{2}{2^{*}-2}}}-\mu b \epsilon^{\alpha} .
\end{aligned}
$$


Since $\alpha<1$ the result follows.

(iii) The proof is similar to the part (ii) and we use the asymptotic estimates (9).

If $Q(x)>0$ on $\bar{\Omega}$, then it is rather difficult to verify that the mountain-pass level is strictly below $S_{\infty, \alpha,-\mu}$. A rudimentary estimate of the mountain-pass level can be obtained with the use of a constant test function. First, we observe that there exists $t_{\circ}>0$ such that

$$
\begin{aligned}
J_{\alpha,-\mu}\left(t_{\circ}\right)=\max _{0 \leq t} J_{\alpha,-\mu}(t) \leq \\
\min \left(\frac{(2-\alpha)(\lambda|\Omega|)^{\frac{2_{\alpha}^{*}}{2_{\alpha}^{\alpha}-2}}}{2(N-2)\left(\mu \int_{\Omega} \frac{d x}{|x|^{\alpha}}\right)^{\frac{2}{2 *}}}-\frac{t_{\circ}^{2^{*}}}{2^{*}} \int_{\Omega} Q(x) d x,\right. \\
\left.\frac{1}{N} \frac{(\lambda|\Omega|)^{\frac{2^{*}}{2^{*}-2}}}{\left(\int_{\Omega} Q(x) d x\right)^{\frac{2}{2^{*}-2}}}-\frac{t_{\circ}^{2_{\alpha}^{*}}}{2_{\alpha}^{*}} \mu \int_{\Omega} \frac{d x}{|x|^{\alpha}}\right) .
\end{aligned}
$$

Using this inequality, for a given interval $[\delta, A], \delta>0$, we can find $\lambda_{\circ}>0$ such that for $0<\lambda \leq \lambda_{\circ}$ an $\delta \leq \mu \leq A$, we have $J_{\alpha,-\mu}\left(t_{\circ}\right)<S_{\infty, \alpha,-\mu}$.

Conversely, given an interval $(0, \Lambda]$ we can find a constant $B>0$, such that for $0<\lambda<\Lambda$ and $\mu>B$, we have $J_{\alpha,-\mu}\left(t_{\circ}\right)<S_{\infty, \alpha,-\mu}$. In both cases we obtain the existence of mountain-pass solutions.

\section{Critical Hardy-Sobolev nonlinearity, case $\mu>0$}

We now consider the following modification of problem (32)

$$
\begin{cases}-\Delta u+\frac{\mu}{|x|^{\alpha}}|u|^{2_{\alpha}^{*}-2} u+\lambda u & =Q(x)|u|^{q-2} u \text { in } \Omega, \\ \frac{\partial}{\partial \nu} u(x) & =0 \text { on } \partial \Omega\end{cases}
$$

with $q \leq 2^{*}$. A variational functional for problem (43) is given by

$$
J_{\alpha, \mu}(u)=\frac{1}{2} \int_{\Omega}\left(|\nabla u|^{2}+\lambda u^{2}\right) d x+\frac{\mu}{2_{\alpha}^{*}} \int_{\Omega} \frac{|u|^{2_{\alpha}^{*}}}{|x|^{\alpha}} d x-\frac{1}{q} \int_{\Omega} Q(x)|u|^{q} d x .
$$

Proposition 8.1. $\quad$ (i) Let $q=2^{*}$. The functional $J_{\alpha, \mu}$ satisfies the $(P S)_{c}$ condition for

$$
c<\min \left(\frac{S^{\frac{N}{2}}}{N Q_{M}^{\frac{N-2}{2}}}, \frac{S^{\frac{N}{2}}}{2 N Q_{m^{\frac{N-2}{2}}}^{2}}\right) .
$$

(ii) If $2<q<2^{*}$, then $J_{\alpha, \mu}$ satisfies the $(P S)_{c}$ condition for every $c$. 
Proof. (i) First we show that a $(P S)_{c}$ sequence $\left\{u_{m}\right\}$ is bounded in $H^{1}(\Omega)$. Indeed, we have

$$
\begin{aligned}
J_{\alpha, \mu}\left(u_{m}\right) & -\frac{1}{2_{\alpha}^{*}}\left\langle J_{\alpha, \mu}^{\prime}\left(u_{m}\right), u_{m}\right\rangle=\left(\frac{1}{2}-\frac{1}{2_{\alpha}^{*}}\right) \int_{\Omega}\left(\left|\nabla u_{m}\right|^{2}+\lambda u_{m}^{2}\right) d x \\
& +\left(\frac{1}{2_{\alpha}^{*}}-\frac{1}{2^{*}}\right) \int_{\Omega} Q(x)|u|^{2^{*}} d x \leq c+1+o\left(\left\|u_{m}\right\|\right)
\end{aligned}
$$

for $m \geq m_{\circ}$. This obviously implies that the sequence $\left\{u_{m}\right\}$ is bounded in $H^{1}(\Omega)$. We can also assume that $u_{m} \rightarrow u$ in $H^{1}(\Omega), L^{2^{*}}(\Omega), L^{2_{\alpha}^{*}}\left(\Omega,|x|^{-\alpha}\right)$. We also have

$$
\left|u_{m}\right|^{2^{*}} \stackrel{*}{\rightarrow}|u|^{2^{*}}+\sum_{j \in J} \nu_{j} \delta_{x_{j}} \text { and }\left|\nabla u_{m}\right|^{2} \stackrel{*}{\rightarrow}|\nabla u|^{2}+\sum_{j \in J} \mu_{j} \delta_{x_{j}}
$$

in the sense of measure. A possible concentration point of $\left\{\frac{\left|u_{m}\right|^{2 *}}{|x|^{\alpha}}\right\}$ is 0 . Using a family of functions concentrating at $x_{j}$ or 0 we derive that

$$
Q\left(x_{j}\right) \nu_{j}=\mu_{j} \text { if } x_{j} \neq 0 \text { and } Q(0) \nu_{\circ}=\mu_{\circ}+\mu \bar{\nu}_{\circ} .
$$

As in the proof of Proposition 2.2 we show that $\nu_{j}=0$ for every $j$ and the result follows.

Since $\left\{u_{m}\right\}$ is bounded in $H^{1}(\Omega)$ we may assume that $u_{m} \rightarrow u$ in $H^{1}(\Omega)$ and $u_{m} \rightarrow u$ in $L k^{q}(\Omega)$. Writing for $n>m$

$$
\begin{aligned}
\left\langle J_{\alpha, \mu}^{\prime}\left(u_{m}\right)-J_{\alpha, \mu}^{\prime}\left(u_{n}\right), u_{m}-u_{n}\right\rangle & =\int_{\Omega}\left(\left|\nabla\left(u_{n}-u_{m}\right)\right|^{2}+\lambda\left(u_{n}-u_{m}\right)^{2}\right) d x \\
& +\mu \int_{\Omega} \frac{\left(\left|u_{m}\right|^{2_{\alpha}^{*}-2} u_{m}-\left|u_{n}\right|^{2_{\alpha}^{*}-2} u_{n}\right)\left(u_{m}-u_{n}\right)}{|x|^{\alpha}} d x \\
& =\int_{\Omega} Q(x)\left(\left|u_{m}\right|^{2^{*}-2} u_{m}-\left|u_{n}\right|^{2^{*}-2} u_{n}\right)\left(u_{n}-u_{m}\right) d x
\end{aligned}
$$

we deduce that $\left\{u_{m}\right\}$ satisfies the Cauchy condition and the result follows.

As a consequence of Proposition 8.1 we can formulate the following existence result.

Proposition 8.2. (i) Let $q=2^{*}, 1<\alpha<2$ and $Q_{M} \leq 2^{\frac{2}{N-2}} Q_{m}$. Suppose that

$$
|Q(y)-Q(x)|=o(|x-y|)
$$

for $x$ near $y$ with $Q(y)=Q_{M}$ and $H(y)>0$. Then for every $\lambda>0$ and $\mu>0$ problem (43) has a solution.

(ii) Let $q=2^{*}$ and $Q_{M}>2^{\frac{2}{N-2}}$. Then there exists $\lambda^{*}>0$ and $\mu^{*}>0$ such that for $0<\lambda \leq \lambda^{*}$ and $0<\mu \leq \mu^{*}$ problem (43) has a solution. 
(iii) Let $2_{\alpha}^{*}<q<2^{*}$. Then there exist $\bar{\lambda}>0$ and $\bar{\mu}>0$ such that for $0<\lambda \leq \bar{\lambda}$ and $0<\mu \leq \bar{\mu}$ problem (43) has a solution.

(iv) Let $2<q<2_{\alpha}^{*}$. Then for every $0<a<b$ there exists $\tilde{\lambda}>0$ such that for every $a \leq \mu \leq b$ and $0<\lambda \leq \tilde{\lambda}$ problem (43) has a solution (this solution is a global minimizer).

Proof. To prove (i), (ii) and (iii) we use the mountain-pass theorem. Part (i) follows by testing $J_{\alpha, \mu}$ with $U_{\epsilon, y}$ and applying the asymptotic estimates (9). Parts (ii) and (iii) follow by testing $J_{\alpha, \mu}$ with a constant function.

(iv) First we show that $J_{\alpha, \mu}$ is bounded from below on $H^{1}(\Omega)$. It follows from the Young inequality that for every $\delta>0$ there exists $C(\delta)>0$ such that

$$
\begin{aligned}
\int_{\Omega} Q(x)|u|^{q} d x & \leq Q_{M}\left(\int_{\Omega} \frac{|u|^{2_{\alpha}^{*}}}{|x|^{\alpha}} d x\right)^{\frac{q}{2 \alpha}}\left(\int_{\Omega}|x|^{\frac{q \alpha}{2_{\alpha}^{2}-q}} d x\right)^{\frac{2_{\alpha}^{*}-q}{2_{\alpha}^{*}}} \\
& \leq \delta \int_{\Omega} \frac{|u|^{2_{\alpha}^{*}}}{|x|^{\alpha}} d x+C(\delta) .
\end{aligned}
$$

Selecting $\delta<\frac{a}{2_{\alpha^{*}}}$ we get

$$
J_{\alpha, \mu}(u) \geq \frac{1}{2} \int_{\Omega}\left(|\nabla u|^{2}\right) d x+\lambda u^{2}+\left(\frac{\mu}{2_{\alpha}^{*}}-\delta\right) \int_{\Omega} \frac{|u|^{2_{\alpha}^{*}}}{|x|^{\alpha}} d x-\frac{C(\delta)}{2^{*}} .
$$

This shows that for every $a \leq \mu \leq b$ and $\lambda>0 J_{\alpha, \mu}$ is bounded from below on $H^{1}(\Omega)$. For $t>0$ we have

$$
J_{\alpha, \mu}(t)=\frac{\lambda t^{2}|\Omega|}{2}+t^{q}\left(\frac{\mu t^{2_{\alpha}^{*}-q}}{2_{\alpha}^{*}} \int_{\Omega} \frac{d x}{|x|^{\alpha}}-\frac{1}{2^{*}} \int_{\Omega} Q(x) d x\right) .
$$

First we choose $t$ so small that

$$
\frac{\mu t^{2_{\alpha}^{*}-q}}{2_{\alpha}^{*}} \int_{\Omega} \frac{d x}{|x|^{\alpha}}-\frac{1}{2^{*}} \int_{\Omega} Q(x) d x<0
$$

for $a \leq \mu \leq b$. Then we choose $\lambda_{*}>0$ small so that $J_{\alpha, \mu}(t)<0$. Hence $\inf _{u \in H^{1}(\Omega)} J_{\alpha, \mu}(u)<0$. The existence of a global minimizer follows from the Ekeland variational principle.

\section{References}

[1] B. Abdellaoui and I. Peral, Some results for semilinear elliptic equations with critical potential, Proc. Roy. Soc. Edinburgh Sect. A 132 (2002), 1-24.

[2] Adimurthi, N. Chaudhuri, and M. Ramaswamy, An improved Hardy-Sobolev inequality and its application, Proc. Amer. Math. Soc. 130 (2002), 489-505 (electronic). 
[3] Adimurthi and G. Mancini, The Neumann problem for elliptic equations with critical nonlinearity, Nonlinear Analysis, Quaderni, Scuola Norm. Sup., Pisa, 1991, pp. 9-25.

[4] _ Geometry and topology of the boundary in the critical Neumann problem, J. Reine Angew. Math. 456 (1994), 1-18.

[5] Adimurthi, G. Mancini, and S. L. Yadava, The role of the mean curvature in semilinear Neumann problem involving critical exponent, Comm. Partial Differential Equations 20 (1995), 591-631.

[6] Adimurthi and K. Sandeep, Existence and non-existence of the first eigenvalue of the perturbed Hardy-Sobolev operator, Proc. Roy. Soc. Edinburgh Sect. A 132 (2002), 1021-1043.

[7] Adimurthi, F. Pacella, and S. L. Yadava, Interaction between the geometry of the boundary and positive solutions of a semilinear Neumann problem with critical nonlinearity, J. Funct. Anal. 113 (1993), 318-350.

[8] Characterization of concentration points and $L^{\infty}$-estimates for solutions of a semilinear Neumann problem involving the critical Sobolev exponent, Differential Integral Equations 8 (1995), 41-68.

[9] Adimurthi and S. L. Yadava, Critical Sobolev exponent problem in $\mathbf{R}^{n}(n \geq 4)$ with Neumann boundary condition, Proc. Indian Acad. Sci. Math. Sci. 100 (1990), 275-284.

[10] H. Brézis and E. Lieb, A relation between pointwise convergence of functions and convergence of functionals, Proc. Amer. Math. Soc. 88 (1983), 486-490.

[11] H. Brézis and L. Nirenberg, Positive solutions of nonlinear elliptic equations involving critical Sobolev exponents, Comm. Pure Appl. Math. 36 (1983), 437-477.

[12] H. Brézis and J. L. Vázquez, Blow-up solutions of some nonlinear elliptic problems, Rev. Mat. Univ. Complut. Madrid 10 (1997), 443-469.

[13] J. Chabrowski, On the nonlinear Neumann problem with indefinite weight and Sobolev critical nonlinearity, Bull. Polish Acad. Sci. Math. 50 (2002), 323-333.

[14] _ Mean curvature and least energy solutions for the critical Neumann problem with weight, Boll. Unione Mat. Ital. Sez. B Artic. Ric. Mat. (8) 5 (2002), 715-733.

[15] J. Chabrowski and M. Willem, Least energy solutions of a critical Neumann problem with a weight, Calc. Var. Partial Differential Equations 15 (2002), 421-431.

[16] N. Chaudhuri and M. Ramaswamy, Existence of positive solutions of some semilinear elliptic equations with singular coefficients, Proc. Roy. Soc. Edinburgh Sect. A 131 (2001), 1275-1295.

[17] H. Egnell, Elliptic boundary value problems with singular coefficients and critical nonlinearities, Indiana Univ. Math. J. 38 (1989), 235-251.

[18] A. Ferrero, Esistenza di soluzioni per equazioni ellittiche singolari a crescita critica, Alessandria, 2000, Tesi di Laurea.

[19] J. F. Escobar, Positive solutions for some semilinear elliptic equations with critical Sobolev exponents, Comm. Pure Appl. Math. 40 (1987), 623-657.

[20] A. Ferrero and F. Gazzola, Existence of solutions for singular critical growth semilinear elliptic equations, J. Differential Equations 177 (2001), 494-522.

[21] J. P. García and I. Peral, Hardy inequalities and some critical elliptic and parabolic problems, J. Differential Equations 144 (1998), 441-476.

[22] D. Gilbarg and N. S. Trudinger, Elliptic partial differential equations of second order, 2nd. ed., Grundlehren der Mathematischen Wissenschaften [Fundamental Principles of Mathematical Sciences], vol. 224, Springer-Verlag, Berlin, 1983, ISBN 3-540-13025-X.

[23] N. Ghoussoub and C. Yuan, Multiple solutions for quasi-linear PDEs involving the critical Sobolev and Hardy exponents, Trans. Amer. Math. Soc. 352 (2000), 5703-5743. 
[24] M. Hoffmann-Ostenhof, T. Hoffmann-Ostenhof, and A. Laptev, A geometrical version of Hardy's inequality, J. Funct. Anal. 189 (2002), 539-548.

[25] P.-L. Lions, The concentration-compactness principle in the calculus of variations. The limit case. I, Rev. Mat. Iberoamericana 1 (1985), 145-201.

[26] P.-L. Lions, F. Pacella, and M. Tricarico, Best constants in Sobolev inequalities for functions vanishing on some part of the boundary and related questions, Indiana Univ. Math. J. 37 (1988), 301-324.

[27] W.-M. Ni, X. B. Pan, and I. Takagi, Singular behavior of least-energy solutions of a semilinear Neumann problem involving critical Sobolev exponents, Duke Math. J. 67 (1992), 1-20.

[28] W.-M. Ni and I. Takagi, On the shape of least-energy solutions to a semilinear Neumann problem, Comm. Pure Appl. Math. 44 (1991), 819-851.

[29] S. Terracini, On positive entire solutions to a class of equations with a singular coefficient and critical exponent, Adv. Differential Equations 1 (1996), 241-264.

[30] J. L. Vázquez and E. Zuazua, The Hardy inequality and the asymptotic behaviour of the heat equation with an inverse-square potential, J. Funct. Anal. 173 (2000), 103-153.

[31] X. J. Wang, Neumann problems of semilinear elliptic equations involving critical Sobolev exponents, J. Differential Equations 93 (1991), 283-310.

[32] Z. Q. Wang, High-energy and multi-peaked solutions for a nonlinear Neumann problem with critical exponents, Proc. Roy. Soc. Edinburgh Sect. A 125 (1995), 1003-1029.

[33] - The effect of the domain geometry on the number of positive solutions of Neumann problems with critical exponents, Differential Integral Equations 8 (1995), 1533-1554.

[34] _ Construction of multi-peaked solutions for a nonlinear Neumann problem with critical exponent in symmetric domains, Nonlinear Anal. 27 (1996), 1281-1306.

[35] _ Existence and nonexistence of G-least energy solutions for a nonlinear Neumann problem with critical exponent in symmetric domains, Calc. Var. Partial Differential Equations 8 (1999), 109-122.

[36] M. Willem, Minimax theorems, Progress in Nonlinear Differential Equations and their Applications, 24, Birkhäuser Boston Inc., Boston, MA, 1996, ISBN 0-8176-3913-6.

[37] M. Zhu, Sharp Sobolev inequalities with interior norms, Calc. Var. Partial Differential Equations 8 (1999), 27-43. 